\title{
ENSAYES SÍSMICOS EN MESA VIBRADORA DE UN EDIFICIO MINIATURA DE 5 NIVELES CON AISLADORES DE BASE
}

\author{
Héctor G. Juárez Ocampo ${ }^{(1)}$, Mario E. Rodríguez ${ }^{(1)}$ y Francisco Gómez Flores ${ }^{(2)}$
}

\begin{abstract}
RESUMEN
En una investigación anterior realizada en mesa vibradora en el Instituto de Ingeniería de la UNAM, se efectuaron ensayes de un edificio miniatura de acero con base fija, sin aisladores, denominado EM2, con un registro de aceleraciones que llevó al espécimen a una respuesta del tipo no lineal. En la investigación que se describe en este artículo, se ensayó un segundo espécimen denominado EM2I, en la misma mesa vibradora y con el mismo registro empleado en la investigación anterior. El espécimen EM2-I, tuvo la misma superestructura que el espécimen EM2, pero con aisladores de base del tipo doble péndulo de fricción. La máxima distorsión de entrepiso medida fue igual a $5.3 \%$ y 0.97\% para los especímenes EM2 y EM2-I, respectivamente. La máxima distorsión global medida fue igual a $4 \%$ y $0.63 \%$ para los especímenes EM2 y EM2-I, respectivamente. El máximo coeficiente sísmico medido en la superestructura aislada fue igual al $26 \%$ del máximo valor medido en el espécimen EM2. Los resultados encontrados en esta investigación muestran la importancia del uso de estructuras aisladas como una solución eficiente para que después de terremotos las estructuras puedan ser ocupadas de manera inmediata.
\end{abstract}

Palabras clave: mesa vibradora; aisladores de doble péndulo de fricción; distorsión de entrepiso; aceleraciones de piso; daños por sismo

\section{SEISMIC TESTS ON SHAKING TABLE OF A MINIATURE BUILDING WITH BASE ISOLATORS}

\begin{abstract}
Shaking table tests were conducted in previous research at the National University of Mexico (UNAM) on a miniature steel building named EM2, using a strong ground motion record. This building represented a conventional building. This paper describes the results of a second test unit called EM2-I, tested in the same shaking table with the same ground motion used in the test of EM2. The test unit with isolators, EM2-I, had a superstructure identical to that of the test unit EM2 but with base isolators of double friction pendulum type. Measured maximum interstory drifts were equal to $5.3 \%$ and $0.97 \%$ for test units EM2 and EM2-I, respectively. Measured maximum global drift was equal to $4 \%$ and $0.63 \%$ for test units EM2 and EM2-I, respectively. The maximum seismic coefficient measured in the isolated superstructure was equal to $26 \%$ of the maximum value for this parameter
\end{abstract}

Artículo recibido el 14 de enero de 2020 y aprobado para su publicación el 6 de junio de 2021. Se aceptarán comentarios y/o discusiones hasta cinco meses después de su publicación.

(1) Instituto de Ingeniería, Universidad Nacional Autónoma de México, Circuito Escolar S/N, Ciudad Universitaria, Del. Coyoacán, 04510, Ciudad de México, e-mail: hector.juarezo@live.com; $\underline{\operatorname{mrod} @ \text { unam.mx }}$

(2) Protección Sísmica de México, 13 Oriente \# 17, Cholula, Puebla, 72760, México, e-mail: contacto@psismicamx.com; fcogof@gmail.com

DOI: $10.18867 /$ ris.105.559 
measured in test unit EM2. Implications of the results in this research are discussed in this paper, showing the importance of using isolated structures as an efficient solution for resisting strong earthquakes and capable of immediate occupancy.

Keywords: shaking table, friction pendulum isolators; interstory drift; floor accelerations; seismic damage

\section{INTRODUCCIÓN}

Diversos reglamentos de construcción en zonas sísmicas del mundo permiten que las estructuras tengan respuesta inelástica sin llegar al colapso durante algún evento sísmico. Esto es, por medio del daño estructural o no estructural se disipa la energía generada por el movimiento del suelo. Esta característica es especialmente relevante cuando se considera la importancia de la estructura y su uso. Para fines de diseño sísmico, la reglamentación actual en Ciudad de México clasifica a las estructuras en grupos y subgrupos (NTCS 2017). Las edificaciones cuya falla estructural podría tener consecuencias graves, pertenecen al grupo A, a su vez, éste se subdivide en los subgrupos A1 y A2. Al subgrupo A1 pertenecen, por ejemplo, las estructuras para hospitales. La característica principal de este tipo de edificaciones es su necesaria operación continua, tanto de equipos como de instalaciones. En general no es posible cumplir con tal requisito sin el empleo de un sistema de protección sísmica. Aunque se logre una estructura bien diseñada y presente daños estructurales menores, la afectación a ocupantes y contenidos, debido a las aceleraciones producidas por terremotos, no podrán ser evitadas, impidiendo su operación continua, como ocurrió con el Hospital Olive View durante el sismo de Northridge de 1994 (U.S. Geological Survey, 1996). El empleo de sistemas de protección sísmica, como es el caso de estructuras con aisladores, permite cumplir tres objetivos, la preservación de la vida de los ocupantes, operación continua y reducción del daño, características de las estructuras resilientes.

El objetivo de la presente investigación fue evaluar experimental y analíticamente la respuesta dinámica de un edificio aislado en su base, y comparar sus resultados con la respuesta dinámica de un edificio a base de marcos convencional con una superestructura idéntica, pero con base fija.

Esta investigación analítica-experimental es la primera de su tipo hecha en Latinoamérica, y busca incentivar futuras investigaciones en el tema, demostrar las ventajas del uso del aislamiento de base y proveer información experimental para posibles revisiones de normas existentes que establezcan los requisitos mínimos de diseño de edificaciones aisladas.

\section{ESTUDIO ANALÍTICO EXPERIMENTAL}

Blandón y Rodríguez (2007), ensayaron en mesa vibradora dos edificios miniatura de acero estructural con el fin de evaluar las fuerzas inerciales en el plano de los sistemas de piso. El término "edificio miniatura" se refiere a que no se emplearon leyes de similitud, por lo que el registro de aceleraciones en la base de la estructura en estudio no fue escalado. En dicha investigación se realizaron ensayes sísmicos de dos edificios de base fija denominados EM1 y EM2. Como resultado, se propuso un procedimiento de diseño sísmico de sistemas de pisos en edificios.

En la presente investigación, se realizaron ensayes sísmicos para estudiar el comportamiento y la respuesta dinámica de un edificio con aisladores en su base. Este edificio se denominó EM2-I, y su superestructura fue idéntica a del espécimen con base fija EM2. Para la realización de los ensayes del 
edificio con aisladores se empleó el mismo registro sísmico utilizado en el estudio de los edificios de base fija. Los aisladores empleados fueron del tipo Doble Péndulo de Fricción, donados por el fabricante FIP Industriale (Italia). A continuación, se describen los edificios convencional y aislado, EM2 y EM2-I, respectivamente.

\section{Espécimen con base fija EM2 (Blandón y Rodríguez, 2007)}

El espécimen EM-2 representa un edificio convencional de base fija con cinco niveles (figura 1), regular en planta, estructurado con base en marcos de acero y sistema de piso formado por seis lingotes soportados por bandejas de acero, atornillados y ligados entre sí por una placa metálica, este conjunto representa la masa de cada nivel (figura 2). Este sistema de piso garantiza un comportamiento de diafragma rígido (Restrepo et al., 2014). La figura 2, muestra el arreglo parabólico de los tornillos que fijan la placa a los lingotes, tal configuración lleva la fuerza inercial a los marcos externos, evitando que las vigas de los marcos transversales, perpendiculares a la dirección del ensaye, se flexionen horizontalmente (Restrepo et $a l ., 2014)$. Las condiciones de apoyo de las bandejas que soportan los lingotes y que descansan sobre las vigas portantes (Vig-Port), ubicadas en el sentido largo, corresponden a las de una viga simplemente apoyada con un extremo que permite el giro y un extremo libre. De este modo, las vigas portantes (figura 1), sólo quedan sometidas a carga gravitacional debida al peso de las bandejas, lingotes y placa. En el sentido corto (figura 1), se encuentran las vigas longitudinales (Vig-Long), cuyo diseño quedó regido por sismo.
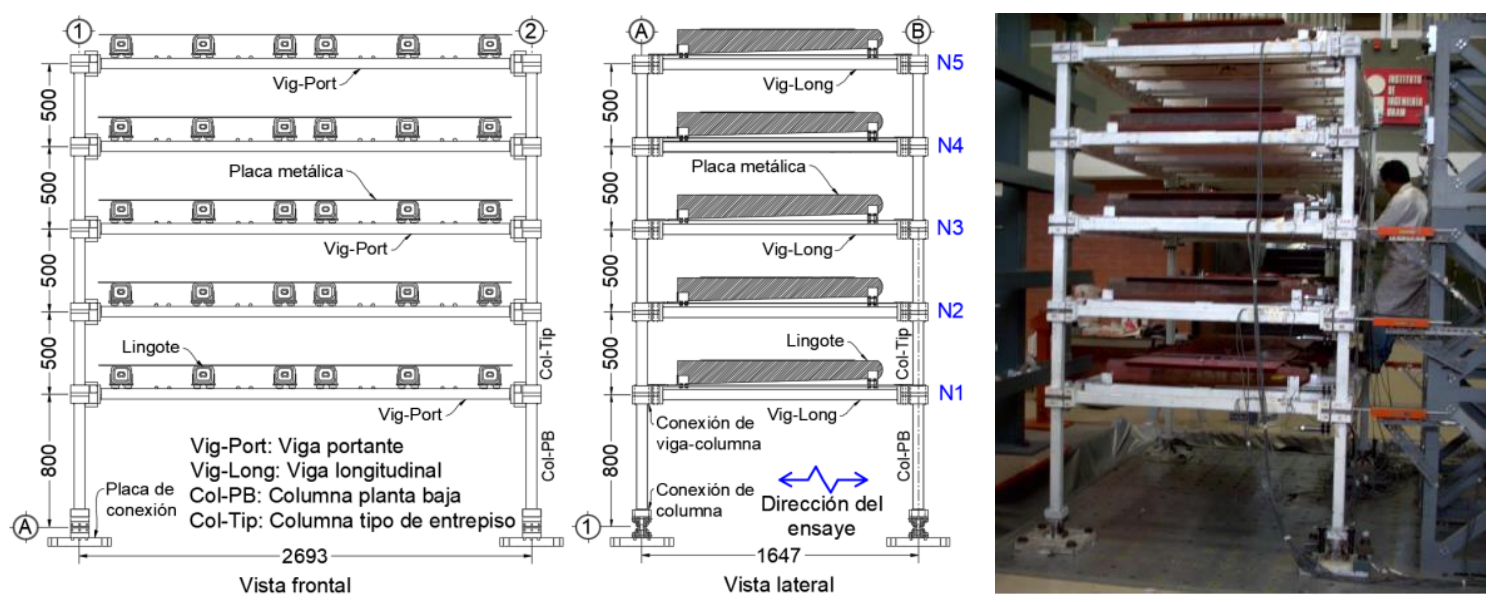

Figura 1. Edificio EM2: vista frontal, lateral e instrumentación (cotas en mm) (Blandón y Rodriguez, 2007)

Las vigas y columnas del espécimen fueron de sección rectangular hueca de 63.5 x 63.5 x $4.5 \mathrm{~mm}$, con esfuerzo de fluencia especificado, $F_{y}$ igual a $3,500 \mathrm{~kg} / \mathrm{cm}^{2}$. La principal característica de este modelo es la incorporación de elementos llamados fusibles, ubicados en la base de columnas de la planta baja (figura 3) y en los extremos de las vigas longitudinales (figura 4), en dichos elementos se concentran las deformaciones inelásticas, permitiendo que el resto de la estructura permanezca elástica. Para estos elementos se empleó acero A36 y sus propiedades mecánicas fueron determinadas por medio de ensayes a tensión de probetas; de estos ensayes se obtuvo un esfuerzo de fluencia igual a $3,060 \mathrm{~kg} / \mathrm{cm}^{2}$. El material empleado para los fusibles fue acero denominado cold rolled. Las vigas y columnas fueron diseñadas para que durante los ensayes se comportaran elásticamente y la respuesta inelástica se deba principalmente a la respuesta de los fusibles. 
Las figuras 3 y 4 muestran el arreglo de placas que forman la conexión placa base-columna y la conexión viga-columna, respectivamente. En ambos casos la conexión fue atornillada. La tabla 1 resume algunas de las características de este edificio. El periodo fundamental y el amortiguamiento fueron obtenidos experimentalmente por Blandón y Rodríguez (2007).

(B)

(A)

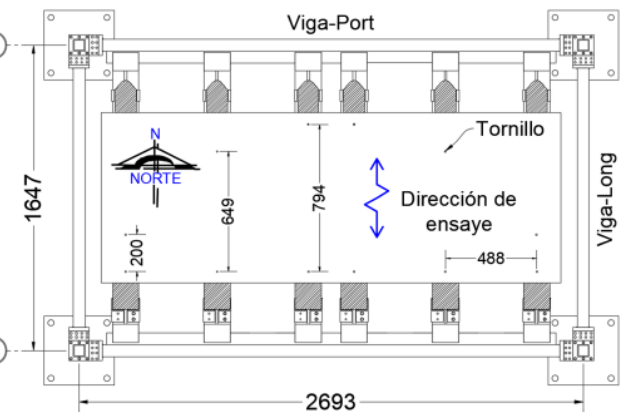

(1)
(2)

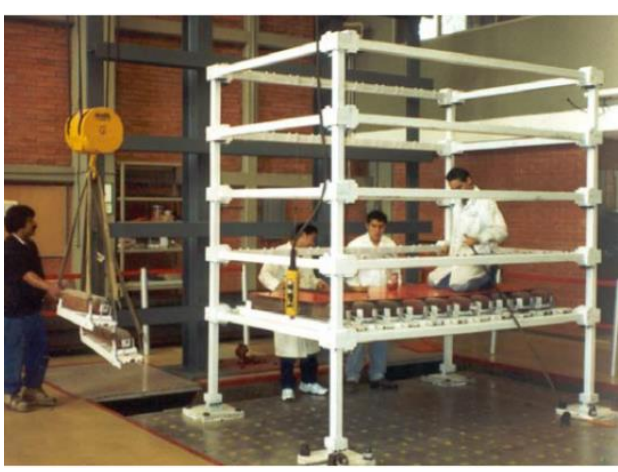

Figura 2. Edificio EM2: planta tipo y montaje del sistema de piso (cotas en mm) (Blandón y Rodriguez, 2007)

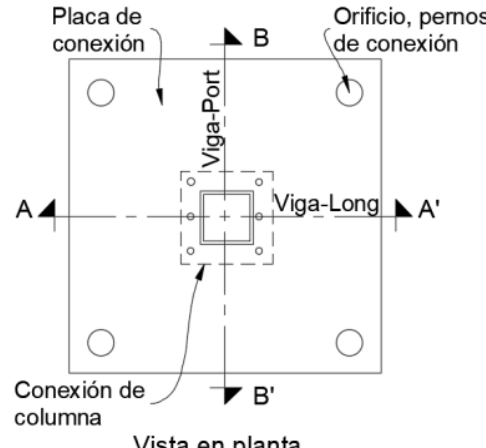

a) columna b)

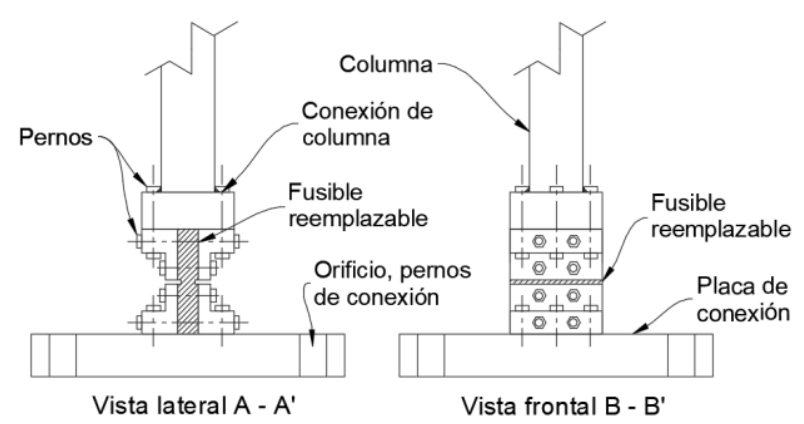

Figura 3. Conexión placa base-columna: a) Planta tipo de conexión, b) Vista lateral y frontal

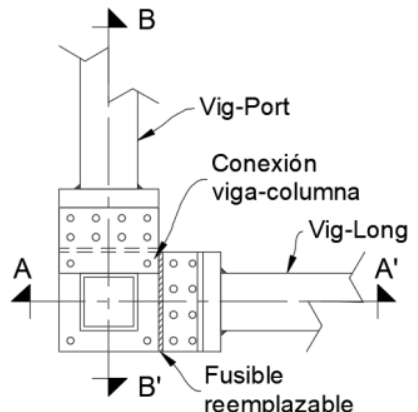

a) Vista en planta

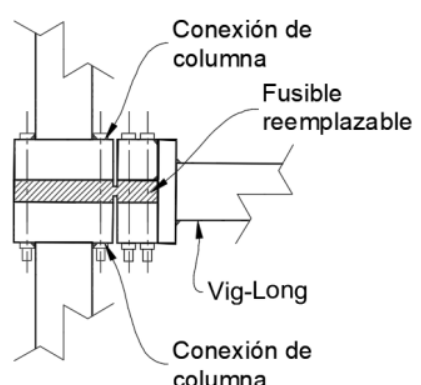

b) Vista lateral $A$ - $A^{\prime}$

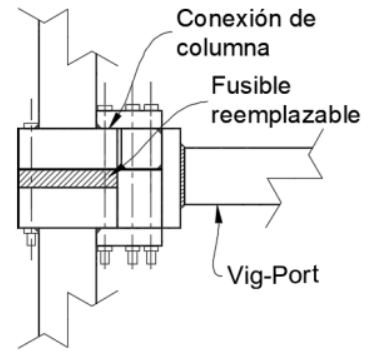

Vista frontal B - B'

Figura 4. Conexión viga-columna: a) Planta tipo de conexión de entrepiso, b) Vista lateral y frontal 
Tabla 1. Características del espécimen EM2

\begin{tabular}{lcrll}
\hline \multicolumn{1}{c}{ Descripción } & Símbolo & & \\
\hline Peso de la superestructura & $\mathbf{W}_{\mathbf{s}}$ & 66.14 & $\mathrm{kN}$ \\
Periodo fundamental & $\mathbf{T}_{\mathbf{1}}$ & 0.485 & $\mathrm{~s}$ \\
Amortiguamiento & $\boldsymbol{\xi}_{\mathrm{s}}$ & $2.0 \mathrm{\%}$ \\
Altura de entrepiso típico & $\mathbf{h}_{\mathbf{i}}$ & $0.50 \mathrm{~m}$ \\
Altura de planta baja & $\mathbf{h P B}_{\mathbf{H}}$ & $0.87 \mathrm{~m}$ \\
Altura total & $\mathbf{H}$ & $2.87 \mathrm{~m}$ \\
\hline
\end{tabular}

\section{Espécimen con aisladores EM2-I}

Este espécimen tenía la misma superestructura que la del espécimen EM2, incluyendo los mismos fusibles, excepto por los aisladores de base, más un nivel de aislamiento identificado como N0 (figura 5). Este nivel tenía las mismas características que los pisos superiores, es decir, la misma masa y distribución de lingotes. Esto permitió comparar las diferencias de la respuesta dinámica de los especímenes con base fija y aislada para la misma demanda sísmica.

Inicialmente se diseñó el espécimen con aislamiento de base empleando aisladores elastoméricos con núcleo de plomo. Sin embargo, las dimensiones que se requerían de estos aisladores eran bastante pequeñas, y no permitieron su fabricación, por lo que se decidió emplear aisladores del tipo péndulo de fricción.
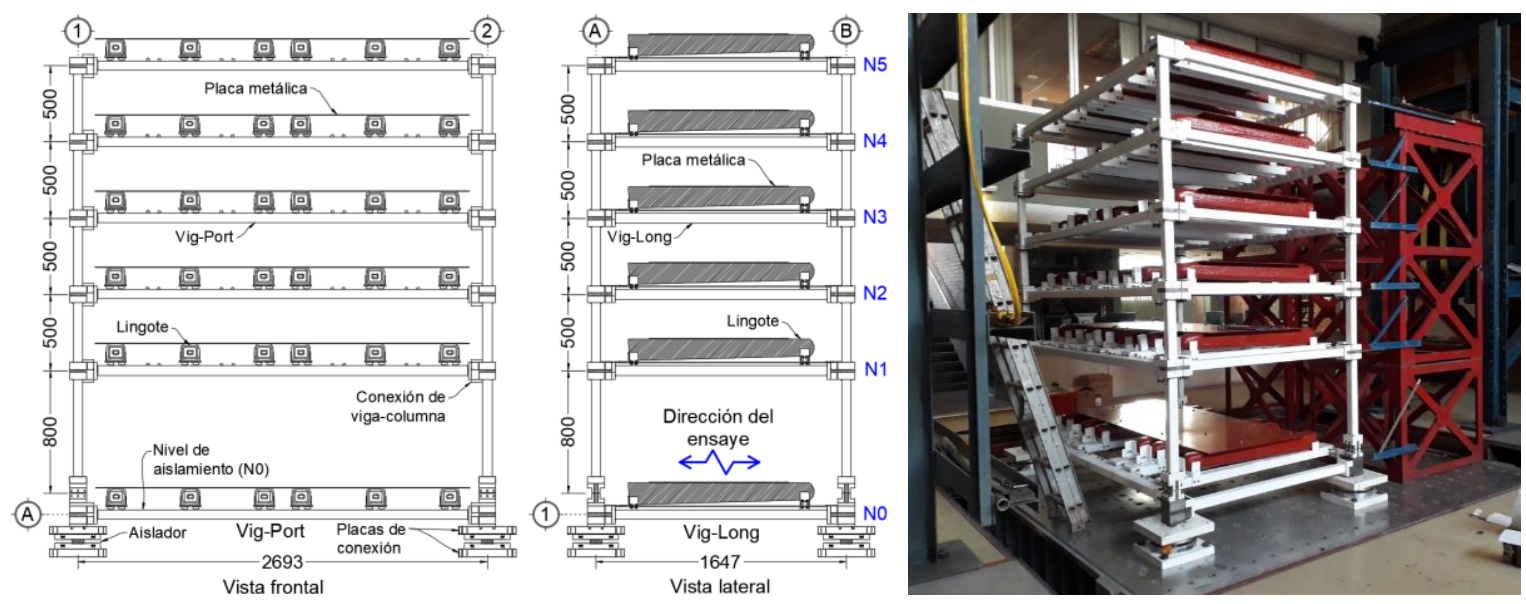

Figura 5. EdificioEM2-I: Vista frontal, lateral e instrumentación (cotas en mm)

El nivel de aislamiento N0 se agregó entre la placa base de la columna y la placa de conexión superior del aislador (figuras 5 y 6). Los aisladores se colocaron por debajo del nivel N0, quedando entre dos placas de conexión, como muestra la figura 6. Las conexiones viga-columna, y columnas de planta baja no requirieron ninguna adaptación y se conservaron como en el edificio EM2 (figura 4). 

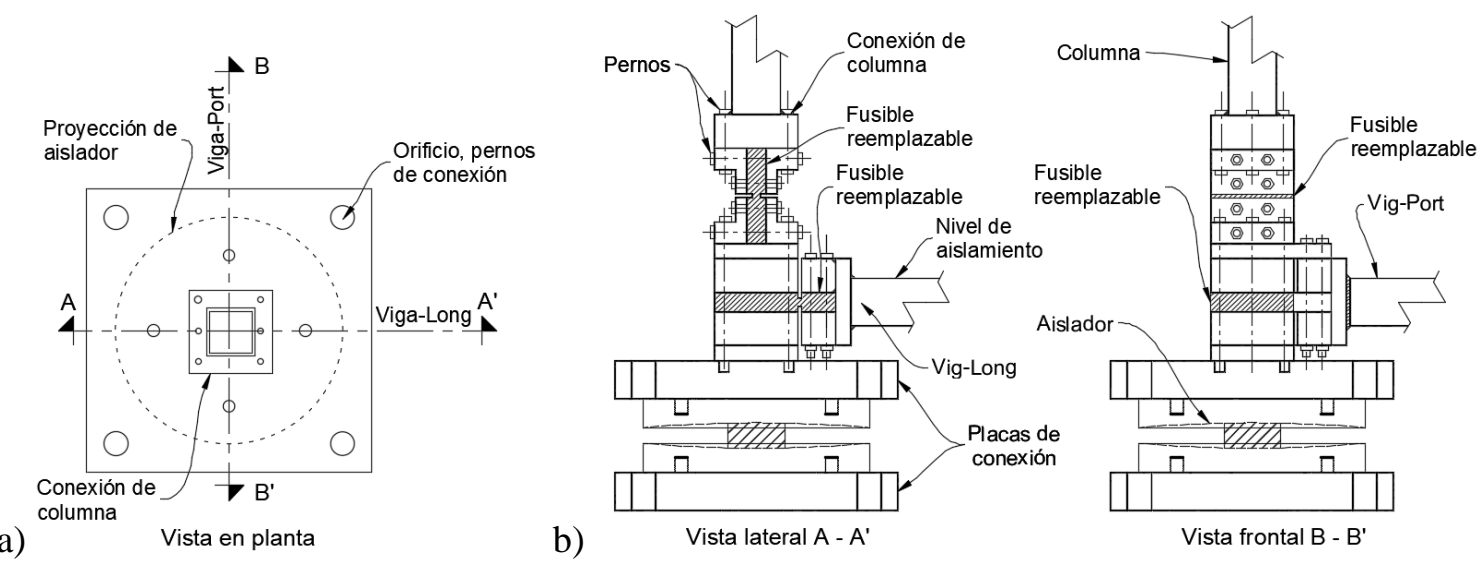

Figura 6. Conexión placa base-aislador-columna: a) Planta tipo de conexión, b) Vista lateral y frontal

La tabla 2 resume algunas de las características del edificio EM2-I. El periodo fundamental y el amortiguamiento, corresponden al valor de desplazamiento máximo, $d_{\text {máx }}$, igual a $176 \mathrm{~mm}$, medido en los ensayes de intensidad alta realizados en esta investigación, que se definen posteriormente.

Tabla 2 Características del edificio EM2-I

\begin{tabular}{lcrl}
\hline \multicolumn{1}{c}{ Descripción } & Símbolo & & \\
\hline Peso de la superestructura & $\mathbf{W}_{\mathbf{s}}$ & $66.14 \mathrm{kN}$ \\
Peso del nivel de aislamiento (N0) & $\mathbf{W}_{\mathbf{N} 0}$ & $13.10 \mathrm{kN}$ \\
Peso total & $\mathbf{W}$ & $79.24 \mathrm{kN}$ \\
Altura de entrepiso típico & $\mathbf{h}_{\mathbf{i}}$ & $0.50 \mathrm{~m}$ \\
Altura de planta baja & $\mathbf{h P B}$ & $0.87 \mathrm{~m}$ \\
Altura total & $\mathbf{H}$ & $3.20 \mathrm{~m}$ \\
Carga axial sobre cada aislador & $\mathbf{N}_{\mathbf{S d}}$ & $19.81 \mathrm{kN}$ \\
Periodo fundamental $\left(d_{\text {máx }}=176 \mathrm{~mm}\right)$ & $\mathbf{T}_{\mathbf{1}}$ & $2.73 \mathrm{~s}$ \\
Amortiguamiento efectivo del aislador $\left(d_{\text {máx }}=176 \mathrm{~mm}\right)$ & $\boldsymbol{\xi}_{\mathbf{e}}$ & $15.10 \%$ \\
\hline
\end{tabular}

Ambos especímenes (EM2 y EM2-I), tuvieron la misma superestructura y representaron edificios miniatura. Por ello, no fue necesario emplear leyes de similitud, que en muchos casos no son representativas del problema físico real. Con base en la hipótesis anterior, no fue necesario escalar el registro empleado en los ensayes.

\section{PROCEDIMIENTO EXPERIMENTAL}

\section{Señal}

Dado que los edificios se supusieron localizados en las costas de Acapulco, Guerrero, se seleccionó un registro de aceleración que tuviera espectro de respuesta con características similares al que se obtendría en dicha zona, y que fuera factible de emplear considerando las limitaciones de desplazamiento de la mesa vibradora. Los sismos en las costas del Pacifico, de acuerdo con la regionalización sísmica presentada en el Manual de Diseño de Obras Civiles de Diseño por Sismo 2015 (MDOC DS, 2015), se encuentran dentro de la franja de alta sismicidad que corresponde a la zona D. Con base en lo anterior y dado que en México no se cuenta con registros de un sismo destructivo con dichas características, se empleó el sismo registrado en 
las costas de Llolleo, ocurrido el 3 de marzo de 1985, en Chile, de $8.0 \mathrm{M}_{\mathrm{W}}$ en la escala de magnitud de momento (7.8 $\mathrm{M}_{\mathrm{S}}$ en la escala de Richter).

\section{Ensayes sísmicos}

Los ensayes sísmicos en la mesa vibradora emplearon una señal digital de aceleraciones del terreno, que aquí se identifica como señal objetivo. A partir de esta señal se generaron dos señales, una de intensidad baja y otra de intensidad alta. Con estas señales se realizaron tres ensayes sísmicos. El primero correspondiente a un sismo de intensidad baja, en el cual las aceleraciones se limitaron al $10 \%$ de los valores de la señal objetivo de intensidad alta. El ensaye y la señal medida en la mesa vibradora durante el ensaye de intensidad baja se identifican como M1-Exp. El segundo ensaye, correspondiente a un sismo de intensidad alta con aceleraciones iguales al $100 \%$ de la señal objetivo, este ensaye y la señal medida se identifican como M2-Exp. Se realizó un tercer ensaye denominado M3-Exp, con las mismas características que el segundo.

Las señales objetivo de intensidad baja y alta, que se utilizaron como señal de entrada en la mesa vibradora, se identificaron como M1-Obj y M2-Obj, respectivamente. La tabla 3 lista los ensayes realizados y las señales objetivo de referencia utilizadas.

Tabla 3. Señales objetivo y señales medidas durante los ensayes del edificio EM2-I

\begin{tabular}{cccc}
\hline Ensaye No. & $\begin{array}{c}\text { Id de señal medida } \\
\text { y ensaye }\end{array}$ & Intensidad & $\begin{array}{c}\text { Señal objetivo de } \\
\text { referencia }\end{array}$ \\
\hline 1 & M1-Exp & $10 \%$ (baja) & M1-Obj \\
2 & M2-Exp & $100 \%$ (alta) & M2-Obj \\
3 & M3-Exp & $100 \%$ (alta) & M2-Obj \\
\hline
\end{tabular}

La figura 7a muestra el acelerograma de la señal M2-Obj y sus valores máximos. La figura 7b muestra el espectro de respuesta de pseudoaceleración elástico de dicha señal, donde se empleó una fracción de amortiguamiento crítico, $\xi$, igual a $3 \%$. Este espectro es característico de terrenos firmes y afecta principalmente a estructuras de periodos $\operatorname{cortos}\left(T_{l}<1 \mathrm{~s}\right)$.

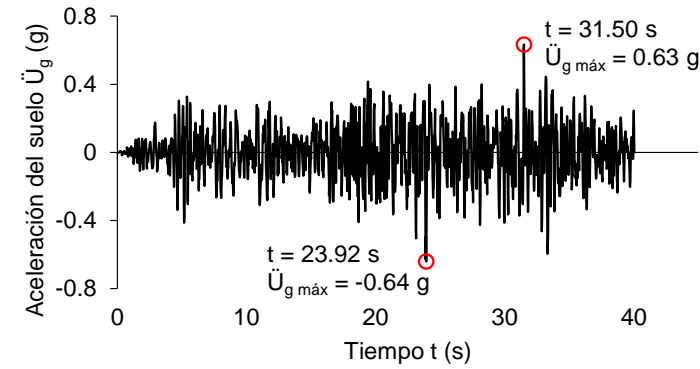

a)

Figura 7. a) Acelerograma (M2-Obj), b) Espectro de respuesta elástico de pseudoaceleración (M2-Obj)

\section{Instrumentación del edificio EM2-I}

Con el fin de conocer los desplazamientos laterales en cada nivel respecto a la mesa vibradora, incluyendo los del sistema de aislamiento, se emplearon dos tipos de dispositivos de medición. El primero, 
constó de transductores de desplazamiento de tipo "Hilo", conocidos así por sus características. Cuentan con un cable delgado de acero que se sujeta en la estructura en estudio y una parte que registra el desplazamiento. Estos dispositivos requieren de una estructura de referencia fija para poder medir el desplazamiento relativo respecto a dicha referencia (figura 8a). Previamente fueron calibrados y por sus características alcanzaron un valor de precisión de $1 \mathrm{~mm}$, valor que se consideró elevado para algunos niveles del espécimen dado los bajos desplazamientos de piso estimados analíticamente. La figura 8a muestra la estructura de referencia y la ubicación de los transductores de hilo. En total se colocaron ocho transductores de este tipo.

El segundo tipo de dispositivo empleado, que tenía mejor precisión que el primer tipo de dispositivo, fue un sistema de medición de coordenadas, para lo cual se empleó una cámara denominada "Optotrak", que es capaz de registrar los desplazamientos en tiempo real con 200 mediciones por segundo, este sistema tiene una precisión de $0.1 \mathrm{~mm}$. Se compone por tres elementos, el equipo adquisidor de datos, la cámara encargada de tomar las lecturas del movimiento en tiempo real (figura 9a) y un conjunto de leds o puntos donde se medían las coordenadas Se obtuvieron mediciones en un total 16 leds colocados en todos los niveles, excepto en el nivel N5, debido al alcance de medición del sistema Optotrak. Se colocó un led en cada placa de conexión de los aisladores y uno al centro de cada fusible (figura 9b).

Para obtener el registro de las aceleraciones de piso y de la mesa vibradora, se colocaron en total 19 acelerómetros, uno sobre la mesa vibradora y tres en cada nivel (A, B y C). La figura 8b muestra una planta tipo con la ubicación y dirección de medición de los acelerómetros instalados.

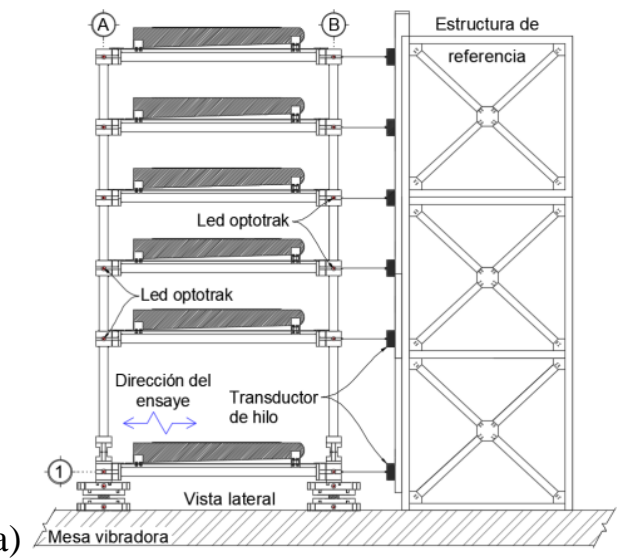

b)

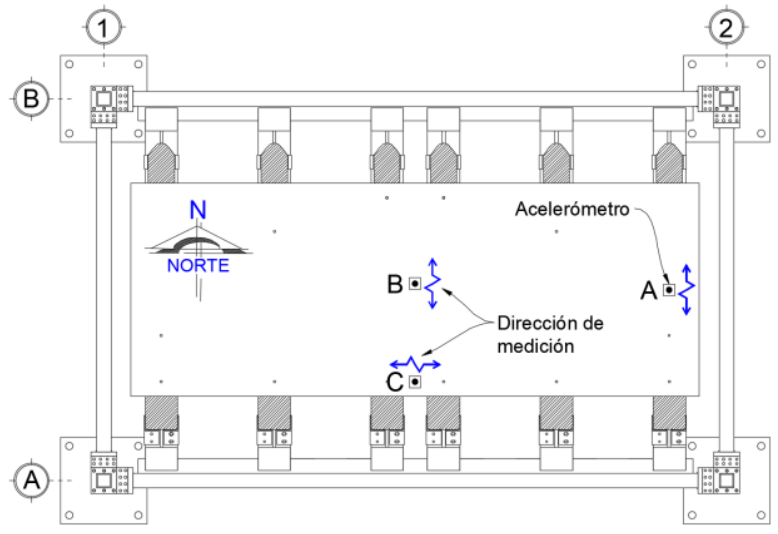

Figura 8. a) Ubicación de transductores de hilo, b) Ubicación y dirección de medición de acelerómetros

La estructura de referencia empleada para fijar los transductores de desplazamiento de tipo "Hilo" fue de acero, con estructuración tipo armadura. Este arreglo ayuda a que esta estructura se desplace igual que la mesa vibradora y pudiera obtenerse la medición de los desplazamientos relativos de piso. Previo a la realización de los ensayes del espécimen, se añadió a la mesa vibradora un peso equivalente al del espécimen, con el objeto de que se pudiera calibrar con los registros objetivos empleados. 
a)

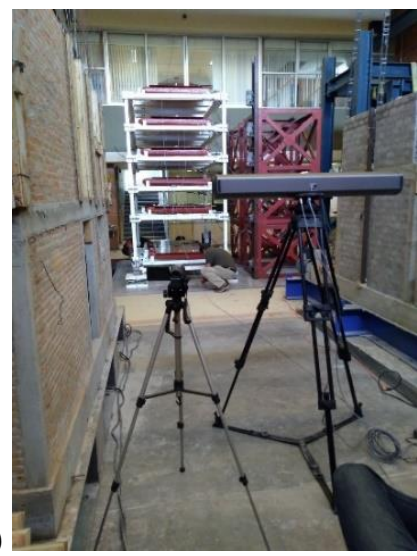

b)

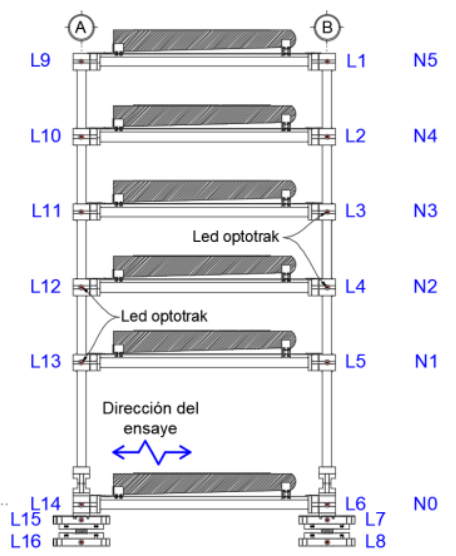

Figura 9. a) Cámara Optotrak, b) Ubicación e identificación de leds (vista lateral)

\section{CARACTERÍSTICAS DE LOS AISLADORES EMPLEADOS}

La respuesta de los aisladores empleados se basa en el principio del péndulo, por lo tanto, el periodo fundamental de una estructura aislada con este sistema dependerá principalmente del radio de curvatura de la superficie curva $R$, y su comportamiento tiende a ser semejante al de un péndulo simple, lo cual brinda independencia de la masa de la estructura. Este tipo de aisladores se componen por tres elementos, dos superficies de deslizamiento curvas y entre ellas un deslizador rígido (figura 10).

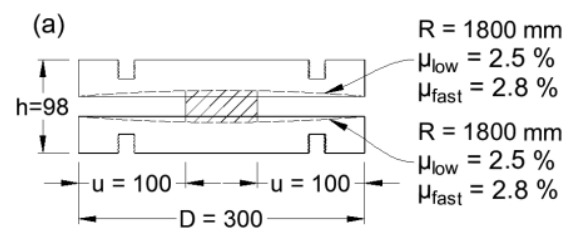

(c)

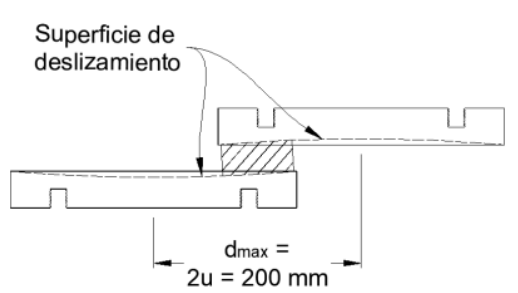

(b)

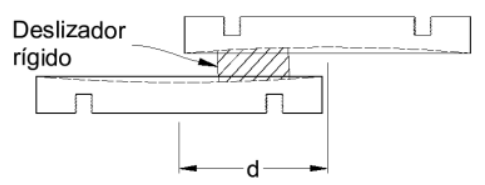

(d)

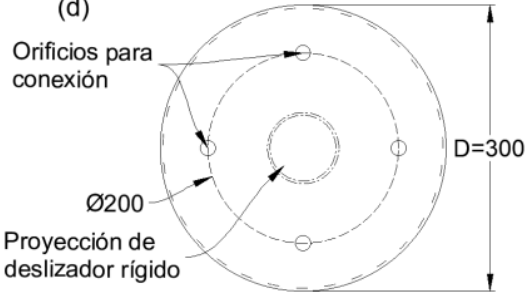

Figura 10. Componentes del sistema de Doble Péndulo de Fricción (cotas en mm)

Las figuras 10a, 10b y 10c, muestran en forma general los elementos que componen a un aislador del tipo Doble Péndulo de Fricción, los que son fabricados empleando acero inoxidable. La figura 10a muestra los radios de curvatura $R$ y valores de los coeficientes de fricción estático, $\mu_{\text {low }} \mathrm{y}$ dinámico, $\mu_{\text {fast }}$, de las superficies de deslizamiento. Los extremos del deslizador rígido están en contacto con las superficies de deslizamiento y se encuentran recubiertos con plástico lubricado. Las dos superficies curvas tienen radio de curvatura $R$ igual a $1800 \mathrm{~mm}$. Los valores de $\mu_{\text {low }}$ y $\mu_{\text {fast }}$ fueron iguales a $2.5 \%$ y $2.8 \%$, respectivamente. Se colocaron en total cuatro aisladores, uno en cada una de las columnas del nivel planta baja del espécimen. Cada una de las superficies inferiores de deslizamiento del aislador, figura 10, se fijó a la mesa vibradora, y las superficies superiores de deslizamiento se fijaron a cada una de las placas base de las columnas del nivel planta baja, lo que se logró empleando cuatro barrenos en cada uno de los elementos curvos (figura 6 y 10d). 
La figura 10b muestra el deslizador rígido, el cual está recubierto con polietileno de ultra alto peso molecular (UHMW-PE, por sus siglas en inglés), alveolado y lubricado. Las dos placas de los aisladores empleados tenían un diámetro $D$, igual a $300 \mathrm{~mm}$, y altura de las dos placas más el aislador, $h$, fue igual a $98 \mathrm{~mm}$ (figura 10a). De acuerdo con el fabricante, los aisladores tenían una máxima capacidad de desplazamiento, $d_{\text {máx }}$, igual a $200 \mathrm{~mm}$ (figura 10c).

\section{Variables que definen el comportamiento no lineal de un aislador}

El comportamiento no lineal de los aisladores de fricción se idealizó mediante la curva bilineal fuerzadesplazamiento que muestra la figura 11 (Naeim y Kelly, 1999). Las variables que definen esta curva son, la fuerza de fricción (ec. 1) antes del desplazamiento, donde $N_{S d}$ es la máxima carga axial que soporta el aislador durante el evento sísmico y $\mu_{\text {slow }}$, es el coeficiente de fricción estático. El parámetro $K_{l}$ (figura 11), es la rigidez inicial, y se define como el cociente entre $F_{o}$ (figura 11) y el desplazamiento de fluencia, $d_{y}$, el cual se supuso igual a $1 \mathrm{~mm}$ (valor recomendado por el fabricante FIP Industriale). El parámetro $K_{2}$, es la rigidez postfluencia, definida por la carga axial y el radio de curvatura $R$ de las superficies de deslizamiento (ec. 2). El parámetro $F_{\text {máx }}$, es la fuerza máxima que puede desarrollar el aislador (ec. 3) y el parámetro $K_{e}$, es la rigidez efectiva (ec. 4). Las ecs. (1) a (4) definen las variables que caracterizan el ciclo histerético que se muestra en la figura 11.

$$
\begin{aligned}
& F_{o}=\mu_{\text {slow }} N_{S d} \\
& K_{2}=\frac{N_{S d}}{R} \\
& F_{\text {máx }}=F_{o}+K_{2} d=\mu_{\text {slow }} N_{S d}+\frac{N_{S d}}{R} d \\
& K_{e}=N_{S d}\left(\frac{1}{R}+\frac{\mu_{\text {slow }}}{d}\right)
\end{aligned}
$$

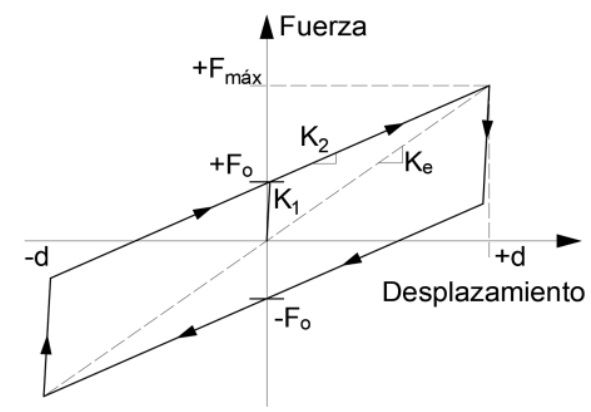

Figura 11. Curva bilineal fuerza-desplazamiento

En las ecuaciones anteriores, $d$ es el desplazamiento horizontal del aislador. Con la ec. 2 y con las definiciones básicas de frecuencia y periodo natural de vibración, es posible obtener la expresión para el cómputo del periodo de vibración del péndulo simple que demuestra su independencia de la masa. 
El coeficiente de fricción $\mu$, es variable y dependiente de la velocidad de desplazamiento, como indica en la ec. 5, (Constantinou et al., 1990). Donde, $v$ es la velocidad de deslizamiento, $\mu_{\text {fast }}$ y $\mu_{\text {slow }}$, son los coeficientes de fricción a una velocidad máxima y mínima, respectivamente. El parámetro $\alpha$ controla el cambio de $\mu_{\text {slow }}$ a $\mu_{\text {sfast }}$. El fabricante de los aisladores (FIP Industriale), determinó experimentalmente los valores de los coeficientes de fricción de acuerdo con European Standard Anti-seismic Devices (EN 15129, 2009). Como resultado, se obtuvo los valores promedio de los coeficientes de fricción $\mu_{\text {slow }} \mathrm{y}$ $\mu_{\text {sfast }}$, los que fueron igual a $2.8 \%$ y $2.5 \%$, respectivamente.

$$
\mu=\mu_{\text {fast }}-\left(\mu_{\text {fast }}-\mu_{\text {slow }}\right) \cdot e^{-\alpha|v|}
$$

Otra característica que mejora la respuesta dinámica de un edificio con aislamiento tipo péndulo de fricción, es el incremento del amortiguamiento generado por la fricción entre el deslizador rígido y las superficies curvas, su valor depende del área dentro del ciclo de histéresis. La ASCE/SEI 7-16 (ASCE/SEI, 2017) define en sus recomendaciones la rigidez efectiva, $K_{e}$ (ec. 6) y el amortiguamiento efectivo, $\xi_{e}$ (ec. 7). En estas ecuaciones se emplean los parámetros $F^{+}$y $\Delta^{+}$, los cuales representan la fuerza y desplazamiento máximos positivos, respectivamente. Los parámetros $F^{-}$y $\Delta^{-}$representan los valores máximos de la fuerza y desplazamiento con valor negativo, respectivamente, de acuerdo con el ciclo de histéresis que desarrolla el aislador. El amortiguamiento efectivo, $\xi_{e}$, es función de Eloop y $K_{e}$, y se determina de acuerdo con la ec. 7.

$$
\begin{aligned}
& K_{e}=\frac{\left|F^{+}\right|+\left|F^{-}\right|}{\left|\Delta^{+}\right|+\left|\Delta^{-}\right|} \\
& \xi_{e}=\frac{2}{\pi}\left(\frac{E_{\text {loop }}}{k_{e}\left(\left|\Delta^{+}\right|+\left|\Delta^{-}\right|\right)^{2}}\right)
\end{aligned}
$$

donde $E_{\text {loop }}$ es el área dentro del ciclo de histéresis en el intervalo $\Delta^{+}$a $\Delta^{-}$(ASCE/SEI, 2017).

\section{MODELOS PARA ANÁLISIS NO LINEAL}

Para estimar la respuesta dinámica del espécimen EM2-I, se llevaron a cabo análisis dinámicos del tipo no lineal (ADNL) empleando dos programas de cómputo, Ruaumoko 2D (Carr, 1998) y ETABS2016 (Computers and Structures, Inc, 2016). El programa Ruaumoko fue diseñado con enfoque a la investigación para realizar análisis del tipo dinámico no lineal de estructuras como edificios o puentes sujetos a terremotos u otro tipo de excitación dinámica. El programa ETABS2016 es ampliamente utilizado por el ingeniero de la práctica y permite la construcción de modelos 3D mediante un ambiente más amigable.

\section{Descripción del modelo analítico en Ruaumoko 2D}

Para la estructura aislada se consideró un total de 76 nodos con tres grados de libertad (figura 12a) y 40 elementos (figura 12b). Los nodos pertenecientes a un mismo nivel se ligaron a un nodo maestro, de esta manera se simplificó a un solo grado de libertad por nivel en la dirección horizontal (dirección del ensaye). 


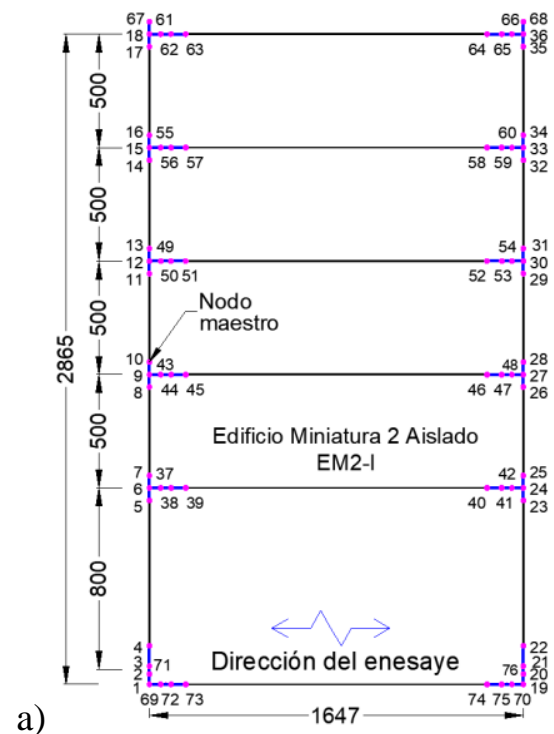

a)

Figura 12. Modelo EM2-I en Ruaumoko 2D. a) Numeración de nodos y b) Numeración de elementos

El programa Ruaumoko emplea el peso de cada nivel concentrada en un nodo maestro (figura 12), el cual en el espécimen fue igual a $6.6 \mathrm{kN}$. En los cálculos de diseño y evaluación de la respuesta de los edificios ante sismos, para la superestructura se consideró una fracción de amortiguamiento crítico, $\xi$, igual a $2 \%$ para los dos primeros modos y $3 \%$ para los modos restantes (Blandón y Rodríguez, 2007). Para el ADNL se empleó la regla de histéresis de Takeda modificada (Carr, 1998), porque no presenta problemas de convergencia.

Los 14 fusibles se modelaron mediante elementos de cuatro nodos, los cuales se caracterizan por generar una liga rígida en los extremos, la cual representa el bloque macizo que fija la placa del fusible. Los nodos internos representan la reducción de las placas de los fusibles, donde se desea inducir el comportamiento inelástico. Las 10 columnas se modelaron con elementos de cuatro nodos y las ligas rígidas representan los bloques de acero en los extremos donde se une cada nivel. Las seis vigas se definieron con elementos de dos nodos, dado que los bloques en los extremos que sirven para fijar la placa de los fusibles ya están considerados.

Para vigas y columnas se utilizaron elementos tipo "Frame" (Carr, 1998), suponiendo para estos elementos una respuesta elástica durante los ensayes. Para los fusibles se utilizaron elementos tipo "Spring" (Carr, 1998), que representa apropiadamente a dichos elementos debido a que el fusible fue diseñado para que forme una articulación plástica en su longitud, no en sus extremos, como se considera en un tipo "Frame" (Restrepo et al., 2014). Para modelar los aisladores se emplearon elementos tipo "Spring". La figura 13 muestra la regla de histéresis empleada y las variables que la define, dichas variables fueron obtenidas a partir de las expresiones anteriormente mostradas.

En la figura $13, \pm F_{y}$, es equivalente a $F_{o}$ (ec. 1 y figura 11 ), y representa la fuerza de fricción inicial que existe en el aislador, quedando definida como la carga axial sobre el aislador por el coeficiente de fricción estático $\mu_{\text {slow }}$ (ec. 1). El parámetro $k_{o}$, es el equivalente a la rigidez inicial $K_{l}$ definida anteriormente (figura 11). El coeficiente $r$, define la rigidez en la etapa no lineal del comportamiento del aislador. 
Una de las desventajas de esta regla de histéresis es que no considera la variación del coeficiente de fricción, valor que depende de la velocidad de desplazamiento (Constantinou et al., 1990). Esta variación en los aisladores empleados en esta investigación fue moderada, por lo que el criterio de ignorar esta variación se considera aceptable de acuerdo con lo observado en estudios similares (Ponzo et al., 2014), donde se concluyó que al emplear valores del coeficiente de fricción constante, $\mu_{\text {slow }}$, en el estudio de los modelos teóricos se obtenían diferencias de alrededor del $10 \%$, respecto a los estudios experimentales.

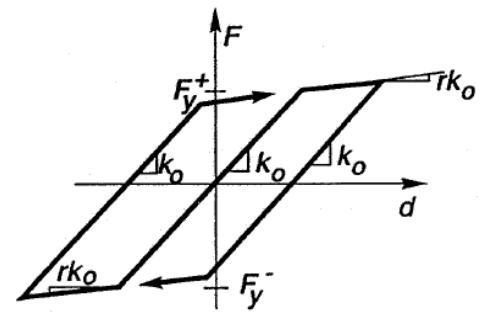

Figura 13. Regla de histéresis bilineal (Ruaumoko 2D)

\section{Descripción del modelo analítico en ETABS2016}

Para la construcción del modelo tridimensional del espécimen EM2-I (figura 14a), se emplearon elementos tipo "Frame" para modelar vigas, columnas, bandejas y elementos de conexión. Para modelar los aisladores se utilizaron elementos tipo "Link" de dos nodos. La asignación de las cargas debidas al peso de los lingotes fue directamente en los nodos de unión de la bandeja con las Vig-Port. Los elementos tipo "Frame" que representan a las bandejas fueron liberados en sus extremos para simular el comportamiento de una viga simplemente apoyada. El análisis no lineal tiempo-historia se realizó empleando el procedimiento definido en ETABS como Análisis No Lineal Rápido (FNA, por sus siglas en inglés), el cual sólo considera la no linealidad de los elementos tipo "Link", en este caso, los aisladores. Para la evaluación de las propiedades dinámicas del edificio, se consideró el peso propio de los elementos más la carga muerta adicional por los lingotes aplicada en los nodos. Para el estudio del comportamiento de los aisladores e empleó el modelo mostrado en la figura 14b, denominado ETABS ISOLATOR2. Este elemento simula el comportamiento bilineal e histerético del aislador de fricción, considerando como datos de entrada los coeficientes $\mu_{\text {slow }}, \mu_{\text {fast }}$, y el parámetro de velocidad $\alpha$, que define la transición entre $\mu_{\text {slow }} \mathrm{y} \mu_{\text {fast }}$, las que se emplean en la ec. 5. El análisis sólo se realizó en dirección del sentido corto, como indica la figura 14a, que es la dirección en la que se desplaza la mesa vibradora.

Las variables mostradas en la figura $14 \mathrm{~b}, C F F 2$ y $C F F 3$, representan al coeficiente $\mu_{\text {fast. }}$. Las variables $C F S 2$ y $C F S 3$ representan al coeficiente $\mu_{\text {slow }}$. Las variables $R 2, R 3$ y $A 2$ y $A 3$, representan al radio de curvatura y el parámetro $\alpha$, respectivamente. La rigidez se identifica con el parámetro $K$. Los números en la figura $14 \mathrm{~b}$ corresponden a los ejes principales. 

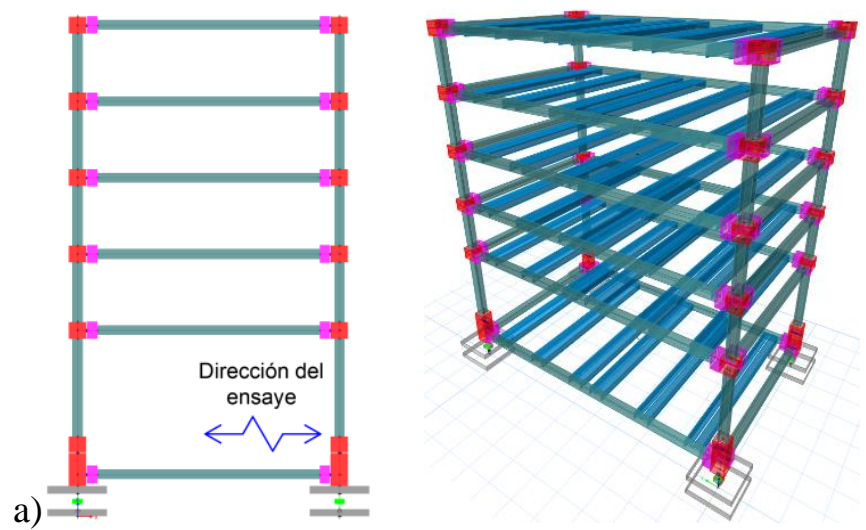

b)

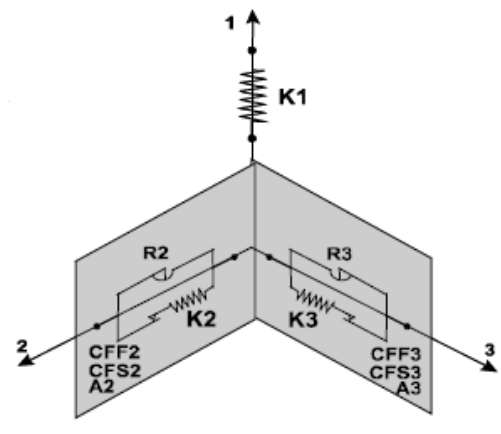

Figura 14. a) Modelo EM2-I en ETABS2016, b) Modelo para idealización del comportamiento de los aisladores

\section{RESULTADOS OBTENIDOS DE LOS ENSAYES}

Se describen los resultados obtenidos durante los tres ensayes realizados, uno de intensidad baja y dos más de intensidad alta. El ensaye de intensidad baja tiene la característica de ser no destructivo y se realizó para determinar las propiedades dinámicas del edificio con comportamiento elástico, verificar que la instrumentación y el sistema adquisidor de datos de la mesa vibradora funcionaran correctamente, y validar los valores de los parámetros empleados en los modelos analíticos elásticos. El ensaye de intensidad alta tiene como objetivo el estudio de la respuesta sísmica del edificio en estudio.

Estos resultados se comparan con los obtenidos analíticamente con Ruaumoko 2D y con ETABS2016. En ambos modelos se emplearon las señales de aceleraciones de la mesa vibradora medidas durante los ensayes. Los resultados analíticos se identifican como muestra la tabla 4, la que indica el orden de los ensayes realizados, la señal empleada para la obtención de la respuesta dinámica y la nomenclatura para hacer referencia a los resultados obtenidos con Ruaumoko 2D (RMK) y ETABS2016 (ETABS).

Tabla 4. Identificación de resultados experimentales y teóricos

\begin{tabular}{cccc}
\hline Ensaye No. & Señal & $\begin{array}{c}\text { Modelo analítico en } \\
\text { Ruaumoko }\end{array}$ & $\begin{array}{c}\text { Modelo analítico en } \\
\text { ETABS2016 }\end{array}$ \\
\hline $\mathbf{1}$ & M1-Exp & RMK (M1-Teo) & ETABS (M1-Teo) \\
$\mathbf{2}$ & M2-Exp & RMK (M2-Teo) & ETABS (M2-Teo) \\
$\mathbf{3}$ & M3-Exp & RMK (M3-Teo) & ETABS (M3-Teo) \\
\hline
\end{tabular}

La determinación de las propiedades dinámicas del edificio EM2-I, se realizó para las dos intensidades de ensayes empleando la información obtenida en los mismos. Como se muestra más adelante, se encontró que la respuesta del espécimen en el ensaye de intensidad baja fue semejante a la de un edificio sin aisladores. Por el contrario, durante el ensaye de intensidad alta, la respuesta del espécimen tuvo características de la respuesta esperada de un edificio aislado.

\section{ENSAYE DE INTENSIDAD BAJA: M1-EXP}

Como se muestra en lo que sigue, se obtuvo la función de transferencia de la señal objetivo respecto a la señal medida, con esta información se determinaron propiedades dinámicas experimentales del espécimen. 


\section{Función de transferencia y espectro de respuesta elástico}

Para corroborar la aproximación de la señal reproducida por la mesa vibradora, se calculó la función de transferencia de la señal objetivo (M1-Obj) respecto a la señal medida (M1-Exp), figura 15a. En estos resultados se observó que la señal es estable hasta aproximadamente valores de frecuencia de $15 \mathrm{~Hz}$, debido a que el ruido de la mesa vibradora afecta a frecuencias mayores a $15 \mathrm{~Hz}$. Este dato es importante dado que el intervalo de frecuencias de los primeros tres modos de vibrar estimados se encuentra entre $1 \mathrm{y} 15 \mathrm{~Hz}$ (tabla 5). La figura 15b, permite comparar la aproximación obtenida entre los espectros de respuesta elásticos de la señal objetivo y la señal medida, en ambos casos se empleó $\xi$ igual a $3 \%$.
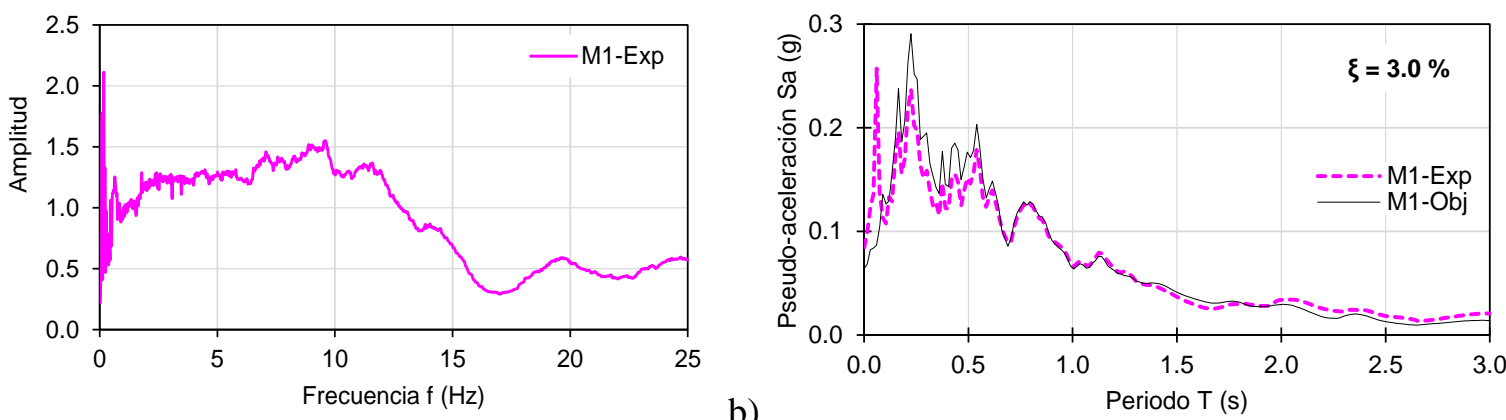

a)

Figura 15. a) Función de transferencia, b) Espectros de respuesta elásticos (ensaye de intensidad baja)

\section{Identificación de las propiedades dinámicas durante el ensaye de intensidad baja}

Para determinar las propiedades dinámicas de la estructura, se calcularon las funciones de transferencia de las aceleraciones medidas en el nivel N5 respecto a las aceleraciones medidas en la base (figura 16), para la obtención de éstas se empleó el programa de computo DEGTRA (Ordaz et al., 2012). Como primer paso se obtuvieron los espectros de Fourier de cada señal, y posteriormente su función de transferencia. Esta función de transferencia se identifica en la figura 16 como M1-exp. Además, la figura 16 muestra las frecuencias obtenidas experimentalmente para el espécimen EM2-I durante los ensayes de intensidad baja. La figura 16 también muestra la función de transferencia de aceleraciones calculadas para el nivel 5 del edificio respecto a las aceleraciones medidas en la base. Estas aceleraciones calculadas se obtuvieron con el programa Ruaumoko 2D. Los resultados de la función de transferencia para este caso se identifican en la figura 16 como M1-Teo. Los parámetros $f_{1}, f_{2}, f_{3}$ y $f_{4}$ corresponden a las frecuencias, éstas están asociadas a los modos $1,2,3$ y 4, respectivamente.

La tabla 5 muestra los resultados experimentales y los resultados analíticos determinados con Ruaumoko 2D empleando la señal medida. Se observa que el periodo fundamental del espécimen EM2-I, obtenido experimentalmente en el ensaye $\mathrm{M} 1-\operatorname{Exp}\left(T_{l}\right.$ igual a $\left.0.58 \mathrm{~s}\right)$, se aproxima al de la estructura de base fija EM2 ( $T_{l}$ igual a $0.485 \mathrm{~s}$ ). Este comportamiento se asocia a la baja intensidad de la señal aplicada, motivo por el cual la fuerza requerida para producir el deslizamiento de los aisladores no fue superada, y, por lo tanto, el sistema de aislamiento no participó en la reducción de la respuesta dinámica. 


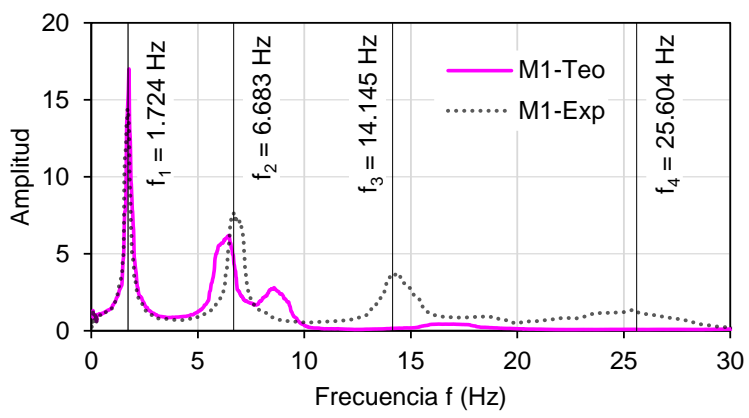

Figura 16. Función de transferencia experimental, M1-Exp y calculada, RMK (M1-Teo)

Tabla 5. Propiedades dinámicas: frecuencias y periodos (ensaye de intensidad baja)

\begin{tabular}{ccccc}
\hline \multirow{2}{*}{ Modo } & \multicolumn{2}{c}{ M1-Exp } & \multicolumn{2}{c}{ RMK (M1-Teo) } \\
\cline { 2 - 5 } & Frecuencia $(\mathbf{H z})$ & Periodo $(\mathbf{s})$ & Frecuencia $(\mathbf{H z})$ & Periodo $(\mathbf{s})$ \\
\hline 1 & 1.724 & 0.580 & 1.724 & 0.580 \\
2 & 6.683 & 0.150 & 6.470 & 0.155 \\
3 & 14.145 & 0.071 & 16.235 & 0.062 \\
4 & 25.604 & 0.039 & 24.871 & 0.040 \\
\hline
\end{tabular}

Un comportamiento característico de los edificios de base fija es la amplificación de las aceleraciones respecto a su altura. Esto se observó en el espécimen EM2-I durante el ensaye de intensidad baja. Este comportamiento se infiere de los resultados mostrados en la figura 17a. Esta figura muestra el registro de aceleraciones medidas en el nivel N5 $\left(\ddot{U}_{5}\right)$, respecto a las aceleraciones medidas en la plataforma de la mesa vibradora $\left(\ddot{U}_{g}\right)$. El cociente de $\ddot{U}_{5}$ y $\ddot{U}_{g}$ fue mayor a uno durante todo el ensaye. Esto demuestra que no hubo atenuación de la aceleración debido la nula participación del sistema de aislamiento. Al final del ensaye $(t$ igual a $40 \mathrm{~s}$ ) se observa que el edificio continúa vibrando libremente. Con esta información fue posible obtener de forma aproximada el valor del periodo estructural durante esta etapa a partir de la definición de periodo natural de vibración, para lo cual se empleó el cociente del tiempo y el número de ciclos completos durante dicho lapso. Con esta aproximación se obtiene $T_{l}$ igual a $0.56 \mathrm{~s}$ (nueve ciclos en $5 \mathrm{~s}$ ), este valor es cercano al determinado mediante la función de transferencia (tabla 5).

Otra evidencia de la nula participación de los aisladores en la respuesta del espécimen EM2-I en el ensaye de intensidad baja, son los bajos desplazamientos relativos de los aisladores medidos entre la placa inferior y superior del aislador. La figura $17 \mathrm{~b}$ muestra la historia de desplazamientos relativos del aislador instrumentado con los leds L7 y L8 (figura 9b). Se obtuvo un desplazamiento máximo de $1.8 \mathrm{~mm}$ en la fase intensa del sismo. Se observa que una vez terminado el ensaye el sistema de aislamiento no regresó a su posición inicial, con un desplazamiento permanente igual a $1.2 \mathrm{~mm}$. El recentrado de los aisladores de este tipo se da en un movimiento sísmico con el peso propio de la superestructura que soportan, esta capacidad es una de las características principales de todos los sistemas de aislamiento. 

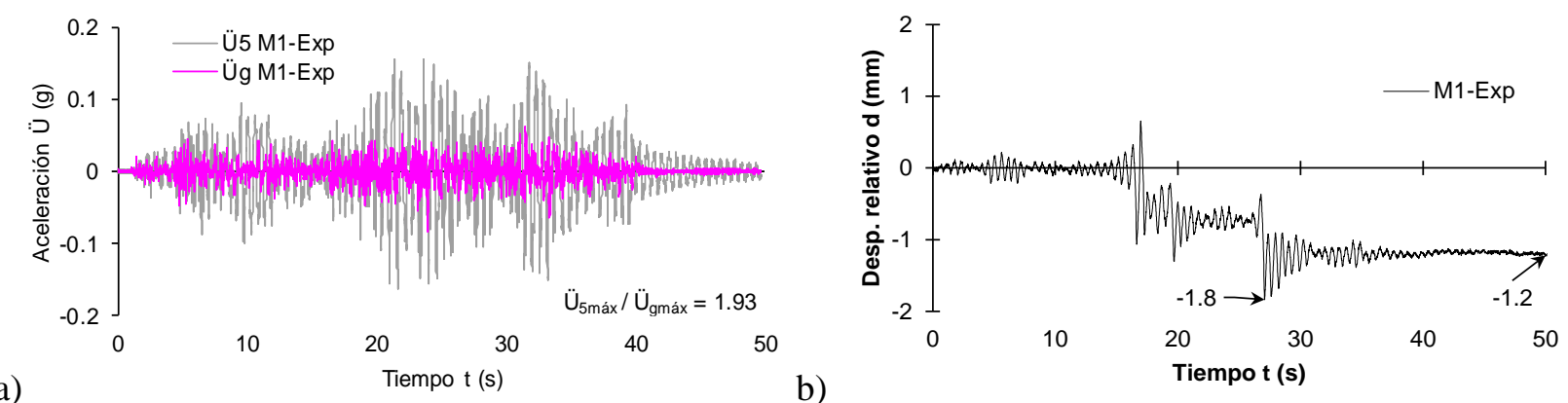

Figura 17. a) Aceleraciones medidas en la plataforma de la mesa vibradora y en el último nivel,

b) Historia de desplazamientos relativos del aislador instrumentado

\section{ENSAYES DE INTENSIDAD ALTA: M2-EXP Y M3-EXP}

La finalidad de estos ensayes fue someter al espécimen EM2-I a las fuerzas sísmicas de diseño, con el fin de obtener la respuesta dinámica del espécimen en estos ensayes, y poder posteriormente compararla con la respuesta estimada analíticamente. Durante estos ensayes se determinaron las propiedades dinámicas. De manera distinta a lo observado en el ensaye de intensidad baja, en el ensaye de intensidad alta se observó el cambio de periodo, pasando del comportamiento como edificio de base fija al comportamiento como edificio de base aislada. El segundo ensaye de intensidad alta, M3-Exp, se realizó con el propósito de corroborar los datos obtenidos durante el ensaye M2-Exp, con el fin de observar el comportamiento del sistema aislado ante dos eventos de intensidad alta consecutivos. En Rodriguez (2020) se puede encontrar videos del ensaye M2-Exp.

\section{Función de transferencia y espectro de respuesta}

La función de transferencia de la señal objetivo respecto a las señales medidas durante los dos ensayes de intensidad alta (figura 18a), y la comparación de los espectros de respuesta de pseudoaceleración elásticos (figura 18b), permitieron verificar el procedimiento de calibración que se realizó a la mesa vibradora. Como se mencionó anteriormente, se identificó que el ruido de la mesa afectó a frecuencias mayores que $15 \mathrm{~Hz}$.

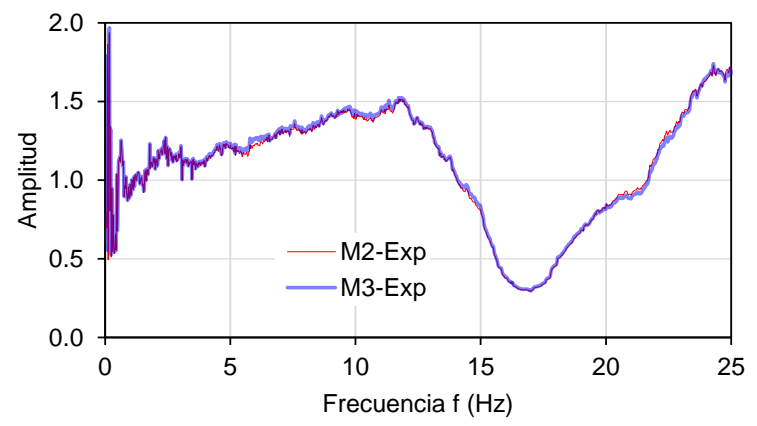

b)

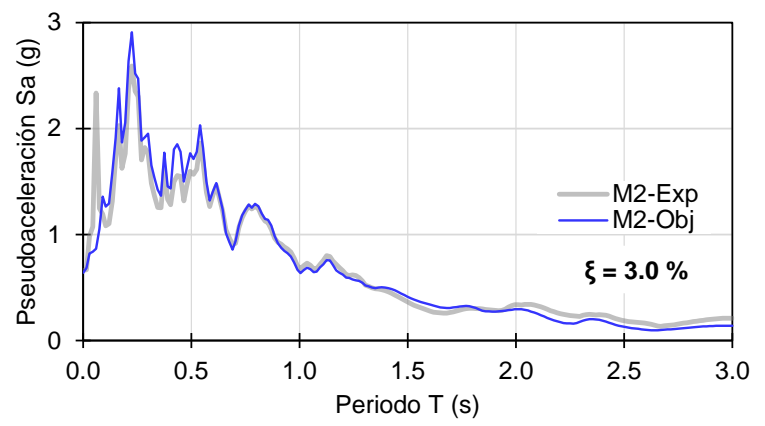

Figura 18. Ensayes de intensidad alta, a) Función de transferencia, b) Espectros de respuesta elásticos

\section{Identificación de las propiedades dinámicas durante los ensayes de intensidad alta}

La obtención de las propiedades dinámicas del edificio sometido a los ensayes de intensidad alta, se hizo siguiendo el mismo procedimiento usado en la prueba de intensidad baja. Para la obtención de los periodos de vibración de la estructura aislada se seleccionó un intervalo de $10 \mathrm{~s}$, correspondiente a la etapa 
de mayor intensidad del sismo, la cual comprende el intervalo 22 a $32 \mathrm{~s}$. La figura 19 muestra los resultados obtenidos de ambos ensayes (M2-Exp y M3-Exp). La tabla 6 muestra las frecuencias y periodos vibración obtenidos en los dos ensayes de intensidad alta. La frecuencia $f_{l}$, corresponde a la frecuencia de los aisladores, y $f_{2}$ representa la frecuencia de la superestructura modificada por la presencia del sistema de aislamiento.

El periodo fundamental de la estructura aislada se pudo estimar empleando la ecuación del periodo de vibración del péndulo simple. Utilizando dicha ecuación se obtuvo para $T_{l}$ el valor $2.691 \mathrm{~s}$, valor bastante cercano al periodo fundamental obtenido experimental y analíticamente. La función de transferencia obtenida y la comparación de los espectros de respuesta respecto a las señales objetivos, presentan una aproximación aceptable, asimismo, las propiedades dinámicas obtenidas analíticamente muestran una buena aproximación respecto a los valores experimentales.
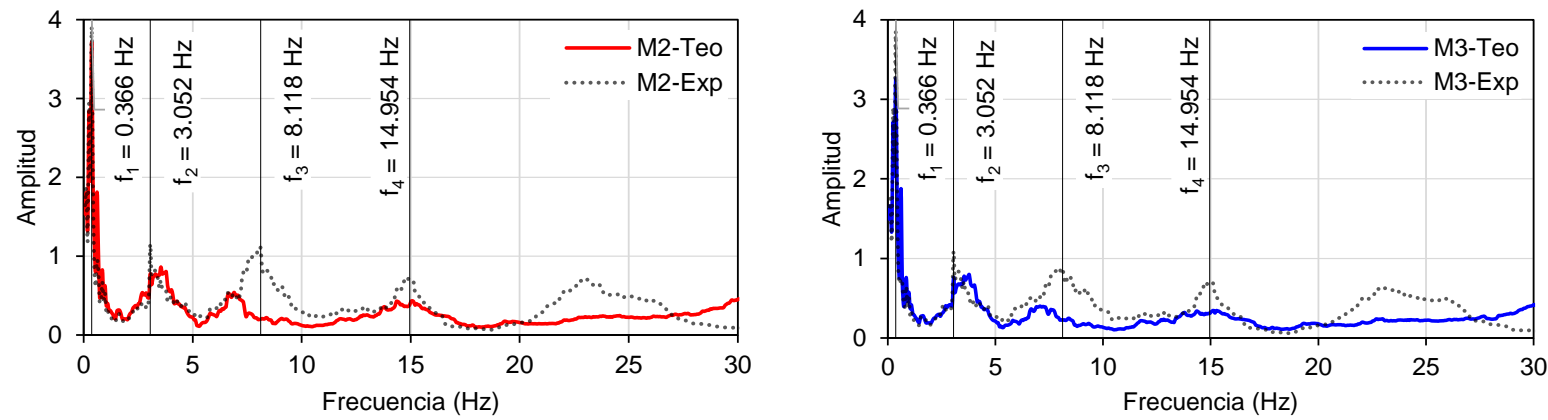

Figura 19. Funciones de transferencia experimental (Exp) y calculadas (Teo)

Tabla 6. Propiedades dinámicas: frecuencias y periodos (ensayes de intensidad alta)

\begin{tabular}{ccccccccc}
\hline \multirow{2}{*}{ Modo } & \multicolumn{2}{c}{ M2-Exp } & \multicolumn{2}{c}{ RMK (M2-Teo) } & \multicolumn{2}{c}{ M3-Exp } & \multicolumn{2}{c}{ RMK (M3-Teo) } \\
\cline { 2 - 9 } & $\begin{array}{c}\text { Frecuencia } \\
(\mathbf{H z})\end{array}$ & $\begin{array}{c}\text { Periodo } \\
(\mathbf{s})\end{array}$ & $\begin{array}{c}\text { Frecuencia } \\
(\mathbf{H z})\end{array}$ & $\begin{array}{c}\text { Periodo } \\
(\mathbf{s})\end{array}$ & $\begin{array}{c}\text { Frecuencia } \\
(\mathbf{H z})\end{array}$ & $\begin{array}{c}\text { Periodo } \\
(\mathbf{s})\end{array}$ & $\begin{array}{c}\text { Frecuencia } \\
(\mathbf{H z})\end{array}$ & $\begin{array}{c}\text { Periodo } \\
(\mathbf{s})\end{array}$ \\
\hline 1 & 0.366 & 2.732 & 0.366 & 2.732 & 0.366 & 2.732 & 0.366 & 2.732 \\
2 & 3.052 & 0.328 & 3.540 & 0.282 & 3.052 & 0.328 & 3.540 & 0.282 \\
3 & 8.118 & 0.123 & 7.141 & 0.140 & 8.118 & 0.123 & 7.141 & 0.140 \\
4 & 14.954 & 0.067 & 15.502 & 0.065 & 14.954 & 0.067 & 14.954 & 0.067 \\
\hline
\end{tabular}

\section{COMPARACIÓN DE RESULTADOS EXPERIMENTALES Y ANALÍTICOS}

Los resultados obtenidos durante los ensayes de intensidad alta se compararon con los resultados analíticos (Ruaumoko 2D y ETABS2016), éstos se identificaron de acuerdo con la nomenclatura que muestra la tabla 4. La predicción de la respuesta dinámica del espécimen EM2-I antes de realizar los ensayes en mesa vibradora se obtuvo empleando las señales objetivo de intensidad baja e intensidad alta, M1-Obj y M2-Obj, respectivamente. Se estimaron aceleraciones y desplazamientos máximos con el fin de poder diseñar los aisladores antes de su fabricación, y para definir las características de la instrumentación a emplear en los ensayes en mesa vibradora. Posterior a los ensayes, se llevó a cabo el cómputo de la respuesta dinámica empleando las señales medidas.

Las figuras 20a y 20b, muestran las envolventes de aceleraciones absolutas de piso medidas y calculadas, expresadas como fracción de la gravedad $\left(\ddot{U}_{i}\right)$. Las aceleraciones calculadas se obtuvieron tanto 
con el programa Ruaumoko como con el programa ETABS. Durante el ensaye M2-Exp, las máximas aceleraciones absolutas medidas en los niveles $\mathrm{N} 1$ y N5, fueron iguales a 0.186 y $0.219 \mathrm{~g}$, respectivamente. En el ensaye M3-Exp se registraron valores de 0.208 y $0.267 \mathrm{~g}$, en los niveles N1 y N5, respectivamente. Estos resultados muestran en el espécimen aislado la atenuación de las aceleraciones absolutas de piso cuando responde a las aceleraciones de la base, lo cual en este caso obedece a un comportamiento de la estructura que tiende a ser del tipo de cuerpo rígido. Las aceleraciones absolutas medidas en el nivel N5 durante los ensayes M2-Exp y M3-Exp, fueron iguales al 33 y $40 \%$ de la máxima aceleración medida en la plataforma de la mesa vibradora, respectivamente. Las máximas aceleraciones de piso se registraron en el nivel N0, $\ddot{U}_{0}$, las que fueron iguales a 0.294 y $0.314 \mathrm{~g}$, en los ensayes M2-Exp y M3-Exp, respectivamente. En el resto de los niveles, los valores de las aceleraciones de piso máximas que se registraron fueron menores que los valores de aceleraciones de piso que ocasionan daño a apéndices y elementos no estructurales. De acuerdo con ATC (2017), se puede considerar que estos valores son mayores que $0.3 \mathrm{~g}$.
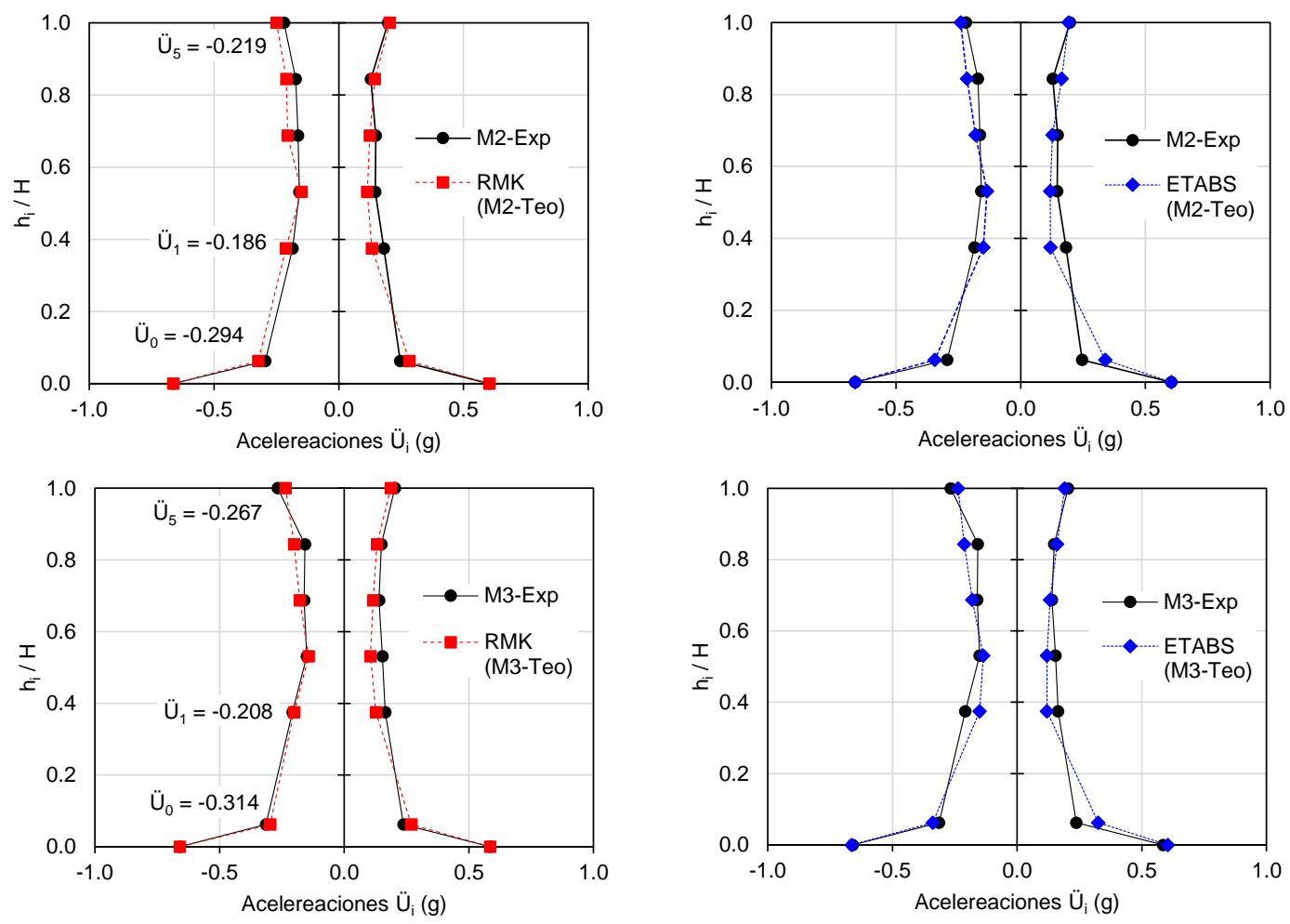

Figura 20. Envolvente de aceleraciones absolutas de piso, a) M2-Exp, b) M3-Exp (ensayes de intensidad alta)

Las figuras 21a y $21 \mathrm{~b}$, muestran las envolventes medidas y calculadas de desplazamientos relativos a la plataforma de la mesa vibradora, $\delta_{i}$, entre la altura del edificio, $H$. Se observa que la concentración de los desplazamientos se presenta en los aisladores, reduciendo la distorsión de entrepiso en la superestructura, $d r$ (ec. 8), definida como la diferencia entre los desplazamientos laterales de los pisos consecutivos que lo delimitan dividida entre la diferencia de elevaciones correspondiente $\left(h_{i}\right)$.

$$
d r=\frac{\delta_{i+1}-\delta_{i}}{h_{i}}
$$


Durante los dos ensayes de intensidad alta los valores máximos de $d r$ fueron iguales $0.97 \%$ y se concentraron en el nivel N1, que corresponde al nivel con mayor altura de entrepiso ( $h_{i}$ igual a $865 \mathrm{~mm}$ ); en los pisos superiores $\left(h_{i}\right.$ igual a $500 \mathrm{~mm}$ ), se obtuvo un valor promedio de $d r$ igual a $0.45 \%$, en ambos ensayes. Estas distorsiones obtenidas fueron menores que el valor de distorsión límite de seguridad contra colapso ( $d r$ igual a $3 \%$ ) especificado para marcos de acero de ductilidad alta en la NTCS (2017). En los niveles superiores al nivel N1, las distorsiones de entrepiso máximas medidas fueron cercanas a uno de los valores límites especificado por la NTCS (2017) para el control de daños ante sismos frecuentes. Se evaluó la distorsión global, definida como el desplazamiento máximo en el último nivel, $\delta_{n}$, divido por la altura del edificio, $H$ (ec. 9). En ambos ensayes de intensidad alta el desplazamiento máximo medido en el nivel N5 respecto al nivel N0, fue de $18 \mathrm{~mm}$, lo cual representa un valor de distorsión global máxima, $D r_{\text {máx }}$, igual a $0.63 \%$.

$$
\operatorname{Dr}=\frac{\delta_{n}}{H}
$$
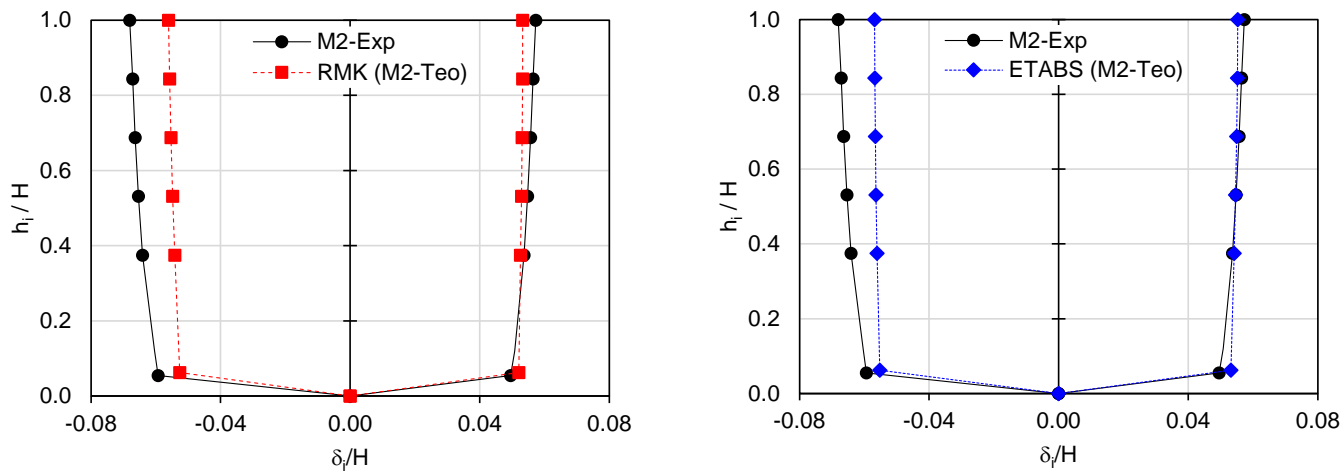

a)
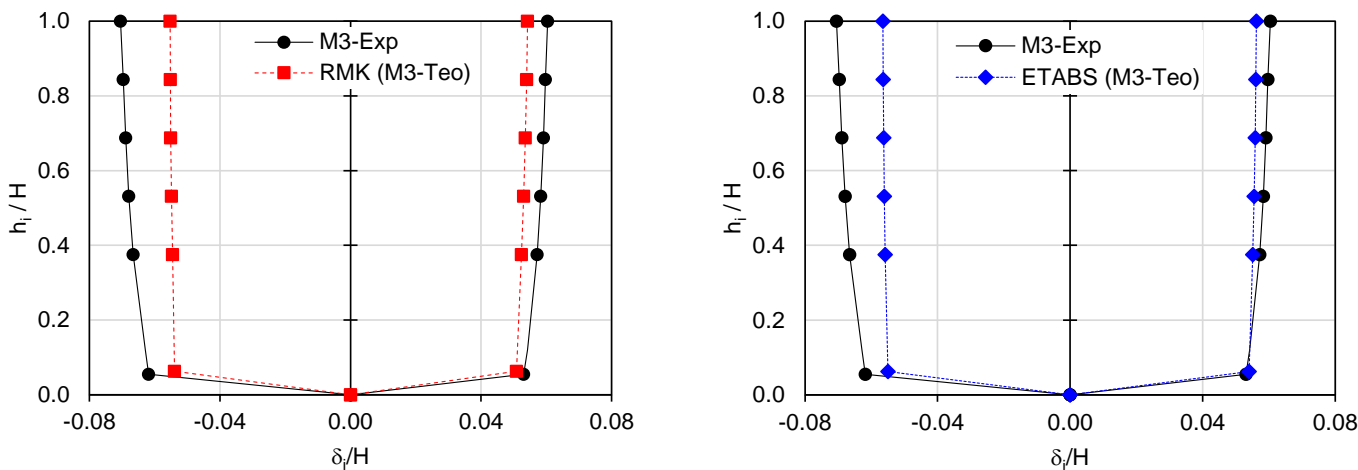

Figura 21. Envolvente de desplazamientos relativos a la plataforma de la mesa vibradora entre la altura del edificio, a) M2-Exp, b) M3-Exp (ensayes de intensidad alta) 
a)
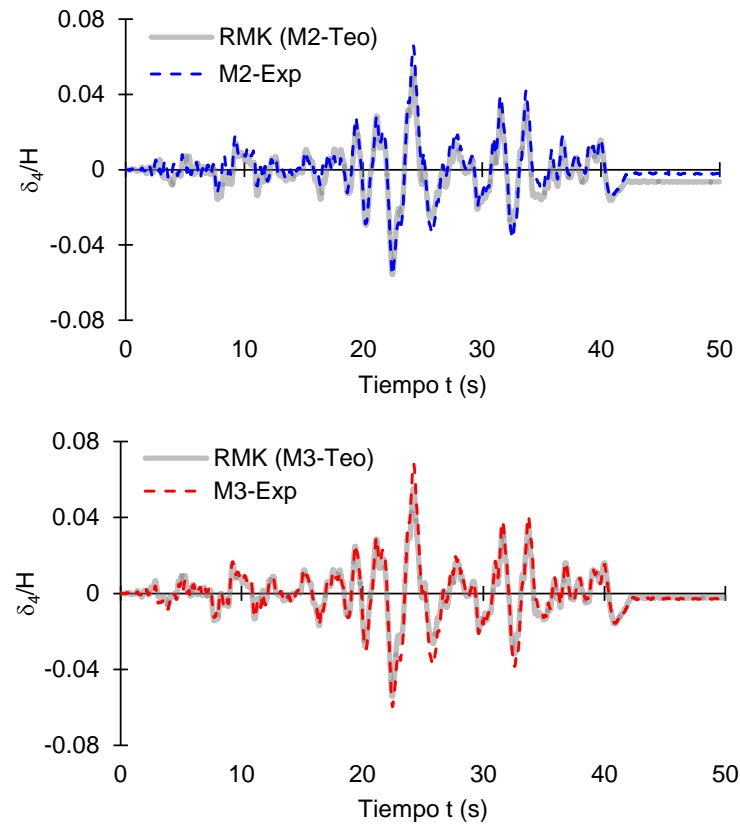
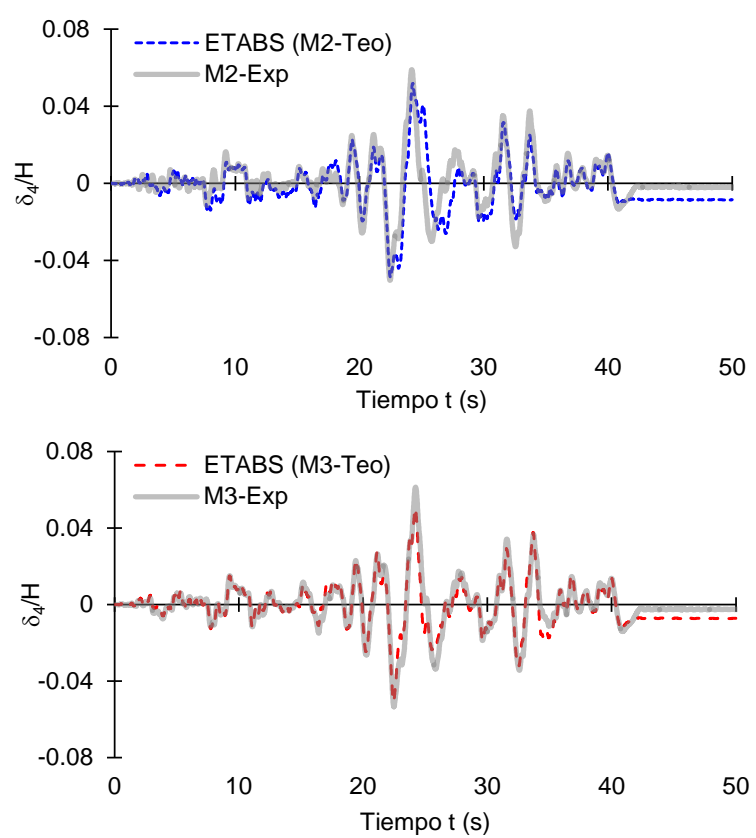

Figura 22. Historia de desplazamientos del nivel N4 relativos a la plataforma de la mesa vibradora entre la altura del edificio. a) Ensaye M2-Exp, b) Ensaye M3-Exp (ensayes de intensidad alta)

Las figuras 22a y 22b, muestran la historia medida y calculada de la relación de desplazamientos del nivel N4 relativos a la plataforma de la mesa vibradora entre la altura del edificio, $H$. Estos desplazamientos se definen como la suma del desplazamiento del nivel en estudio respecto al nivel N0 y los desplazamientos de los aisladores. En el nivel N5 del espécimen EM2-I el desplazamiento máximo fue igual a 190 mm y 198 mm en los ensayes M2-Exp y M3-Exp, respectivamente.

Los resultados del cómputo de aceleraciones y desplazamientos obtenidos del ADNL empleando Ruaumoko 2D y ETABS2016, muestran una correlación aceptable con los resultados experimentales.

\section{Comportamiento histerético de los aisladores}

La disipación de energía de los aisladores de este tipo se debe a la fricción entre las superficies de deslizamiento y el deslizador rígido. Las figuras 23a y 23b, muestran los ciclos de histéresis (teórico y experimental), desarrollados durante el comportamiento no lineal de los aisladores en los ensayes M2-Exp y M3-Exp, respectivamente. Estas figuras además muestran los valores máximos medidos del cortante basal y de desplazamientos respecto al nivel de la mesa vibradora. Las ordenadas fueron normalizadas por la máxima fuerza cortante medida en los aisladores, $V_{\text {máx EXP. }}$. Esta fuerza cortante fue igual a $2.35 \mathrm{kN}$ para el ensaye M2-Exp, y fue igual a $2.38 \mathrm{kN}$ para el ensaye M3-Exp.

La figura 23 muestra una característica importante de los lazos de histéresis del aislador, los cuales no presentan degradación de rigidez en los ciclos de carga. Al comparar el comportamiento histerético desarrollado por los aisladores durante los ensayes M2-Exp y M3-Exp, se observa mayor estabilidad en los ciclos de histéresis durante el segundo ensaye de intensidad alta, en cambio, durante M2-Exp, la figura 23a muestra ciclos irregulares en la parte inicial del sismo. La máxima fuerza lateral, $F_{\text {máx }}$, desarrollada por los aisladores durante la fase inicial del ensaye M2-Exp, asociada al desplazamiento de fluencia $d_{y}$, fue igual a $2.24 \mathrm{kN}$. Con este valor y la ec. 1, el valor estimado del coeficiente de fricción $\mu_{\text {slow }}$ fue igual a $11.3 \%$. Durante la fase inicial del ensaye M3-Exp, y para el desplazamiento de fluencia $d_{y}$, se obtuvo $F_{\text {máx }}$ igual a 
$0.75 \mathrm{kN}$, con estos valores se estimó un coeficiente de fricción $\mu_{\text {slow }}$ igual a $3.8 \%$. Lo anterior muestra que el valor obtenido para $\mu_{\text {slow }}$ en el ensaye M2-Exp fue alrededor de tres veces el valor de $\mu_{\text {slow }}$ obtenido en el ensaye M3-Exp. Este comportamiento se asocia al material lubricante del deslizador rígido, que por su exposición a la intemperie sufrió un efecto de endurecimiento. Posterior al primer ensaye de intensidad alta, el aislador recuperó sus propiedades con material lubricante.

El programa Ruaumoko 2D no permite utilizar explícitamente las variables que definen el coeficiente de fricción en condiciones dinámicas, y sólo es posible introducirlo de forma indirecta mediante la fuerza de fricción $F_{o}$. El comportamiento se define por la rigidez inicial $k_{o}$, asociada al valor de $F_{o}$ que produce el desplazamiento de fluencia $d_{y}$, y por la rigidez post-fluencia definida como $r k_{o}$, donde $r$ es el factor que describe la pendiente en la etapa no lineal (figura 13). Por estos motivos los ciclos obtenidos son estables y bien definidos sin mostrar variaciones por efecto de la velocidad de carga y presión por la carga sobre los aisladores (figura 23). A pesar de esta limitación del modelo analítico con el programa Ruaumoko, se logró simular aceptablemente el comportamiento de la estructura aislada (figura 23).
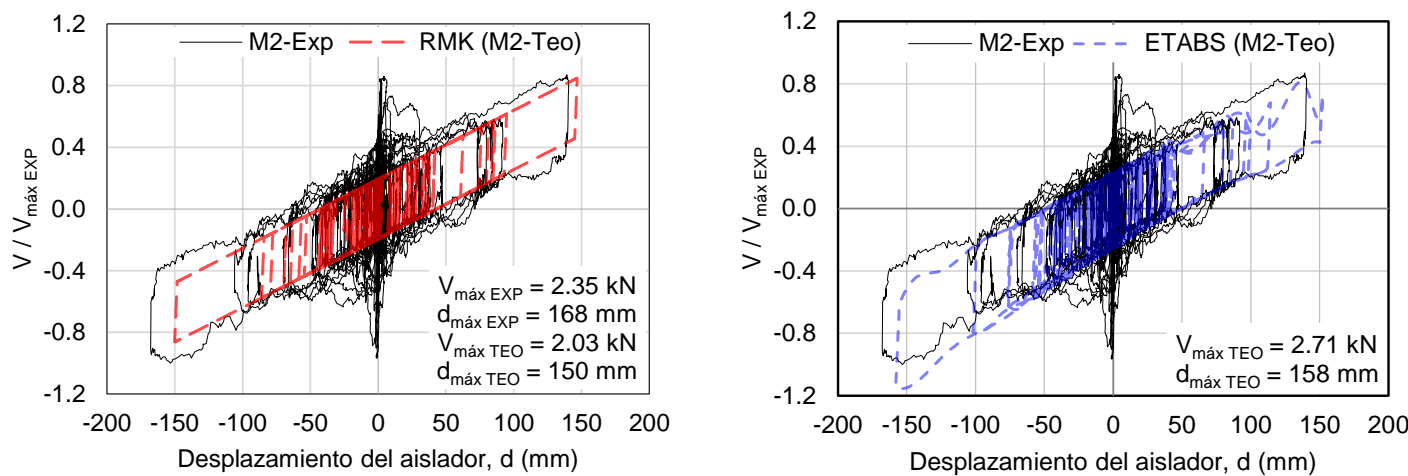

a)
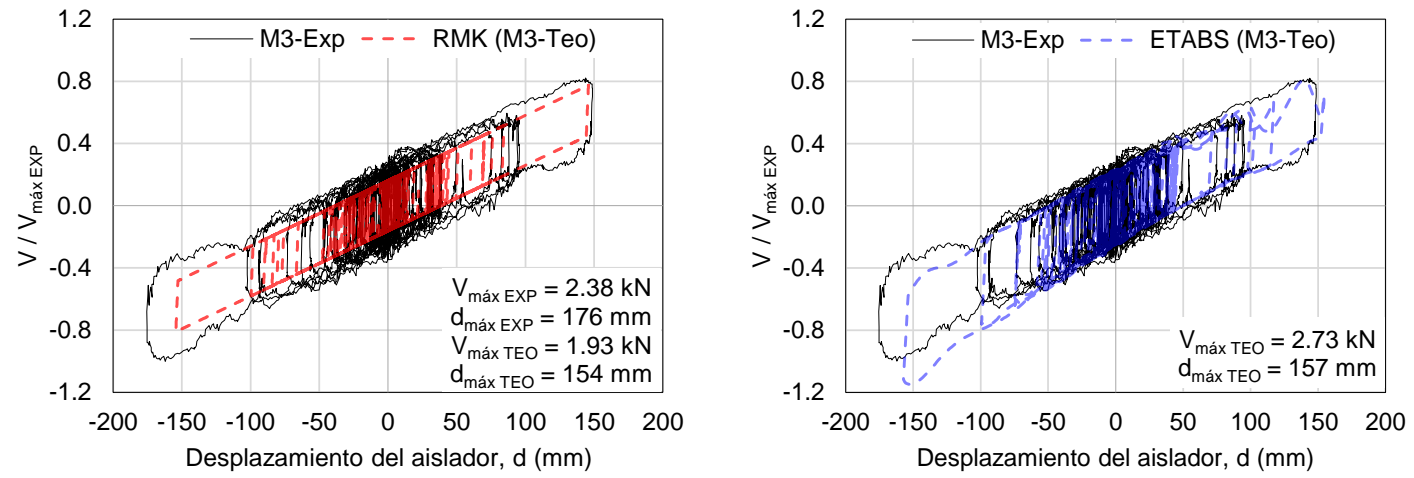

Figura 23. Lazos de histéresis de un aislador durante los ensayes de intensidad alta: a) M2-Exp, b) M3-Exp

Entre las variables que modifican las propiedades de los aisladores de fricción se encuentran, la carga vertical, temperatura, calentamiento de las superficies, envejecimiento, velocidad de carga, superficies con y sin lubricación, entre otros (McVitty y Constantinou, 2015). El modelo de los aisladores en ETABS2016 permitió introducir las variables y propiedades no lineales que describen el comportamiento de éstos en condiciones dinámicas. Se emplearon los coeficientes de fricción $\mu_{\text {slow }}, \mu_{\text {fast }}$, el parámetro de velocidad $\alpha$, el radio de curvatura $R$, la rigidez lateral y la fuerza lateral. Por tal motivo, los ciclos de histéresis obtenidos 
con el programa ETABS muestran un comportamiento con una mejor aproximación a los resultados experimentales.

El efecto del endurecimiento del lubricante sólo afectó la parte inicial del ensaye M2-Exp, incrementando el valor del coeficiente $\mu_{\text {slow }}$. Una vez vencida la fuerza de fricción correspondiente a este coeficiente, se alcanzaron valores de $F_{\text {máx }}$ y $d_{\operatorname{máx}}$ muy similares en ambos ensayes de intensidad alta (figura 23).

\section{Amortiguamiento}

En la literatura se considera que el amortiguamiento es uno de los factores que contribuyen a la capacidad de un sistema para disipar la energía introducida por un movimiento sísmico. Para el caso de los aisladores empleados, el amortiguamiento se produce por el efecto del roce de las superficies curvas y el deslizador rígido, y se relaciona con el área de los ciclos de histéresis. El amortiguamiento efectivo, $\xi_{e}$, se determinó empleando las expresiones recomendadas en la ASCE/SEI 16 (2017) y las variables obtenidas experimentalmente, procedimiento que se resume en lo que sigue.

Fuerza lateral máxima positiva

Fuerza lateral máxima negativa

Desplazamiento máximo positivo

Desplazamiento máximo negativo

Área del ciclo de histéresis

Rigidez efectiva (ec. 6)

Amortiguamiento efectivo (ec. 7)

\section{Ensaye M2-Exp:}

$\mathrm{F}^{+}=2.04 \mathrm{kN}$

$\mathrm{F}^{-}=2.35 \mathrm{kN}$

$\Delta^{+}=140.6 \mathrm{~mm}$

$\Delta^{-}=167.8 \mathrm{~mm}$

$E_{\text {loop }} \approx 4\left(\Delta F_{y}-F \Delta_{y}\right)=323.4$

$K_{e}=\frac{\left|F^{+}\right|+\left|F^{-}\right|}{\left|\Delta^{+}\right|+\left|\Delta^{-}\right|}=14.3 \mathrm{kN} / \mathrm{m}$

$\xi_{e}=\frac{2}{\pi}\left(\frac{E_{\text {loop }}}{k_{e}\left(\left|\Delta^{+}\right|+\left|\Delta^{-}\right|\right)^{2}}\right)=15.1 \%$

\section{Ensaye M3-Exp:}

$\mathrm{F}^{+}=1.95 \mathrm{kN}$

$\mathrm{F}^{-}=2.38 \mathrm{kN}$

$\Delta^{+}=148.7 \mathrm{~mm}$

$\Delta^{-}=175.5 \mathrm{~mm}$

\section{Cortante basal}

El cortante basal, $V_{b}$, en el espécimen se determinó como la sumatoria de las fuerzas inerciales de piso definidas como el producto de los pesos de cada nivel, $W_{i}$, de la superestructura y su aceleración en el tiempo, $\ddot{U}_{i}(t)$, expresada como fracción de la gravedad, como indica la ec. 10. Estas fuerzas inerciales se obtuvieron con el mismo procedimiento que se emplea en estructuras de base fija, considerando todos los pesos de los niveles sobre el nivel de desplante (superestructura), como muestra la figura 24.

$$
V_{b}(t)=\sum_{i=1}^{5} W_{i} \cdot \ddot{U}_{i}(t)
$$

La tabla 7 muestra los máximos valores de $V_{b}(t)$ obtenidos teórica y experimentalmente durante los tres ensayes. Se observa que el valor del coeficiente sísmico máximo, $c_{\text {máx }}$, medido en el ensaye de intensidad baja M1-Exp, fue igual al 84 y $93 \%$ de los valores máximos medidos en los ensayes de intensidad alta M2Exp y M3-Exp, respectivamente. El que los cortantes máximos medidos en los ensayes de intensidad alta y baja tuvieran valores cercanos muestra una importante participación del sistema de aislamiento en la mejora de la respuesta dinámica durante los ensayes de intensidad alta. Esto refleja el efecto del incremento del 
periodo fundamental en la estructura con aisladores, y el incremento del amortiguamiento, respecto a estos parámetros en la estructura con base fija.
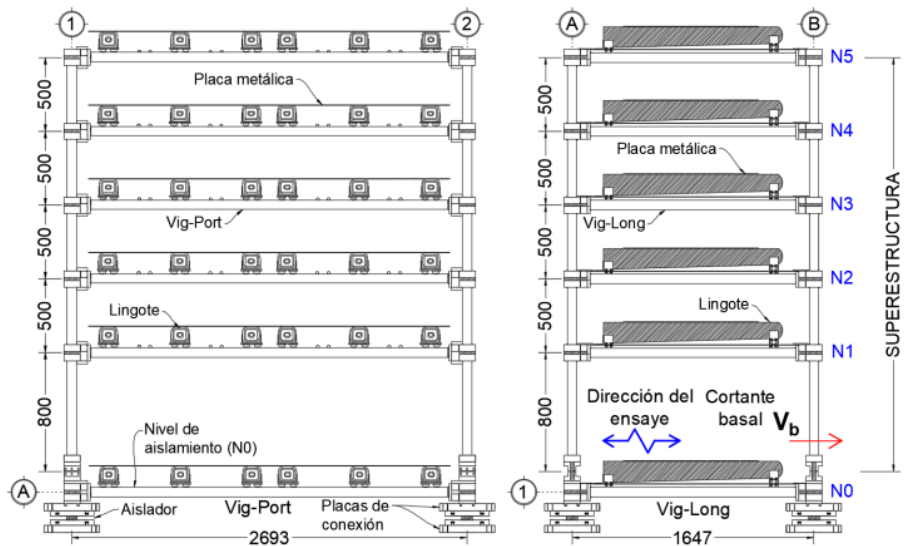

Figura 24. Cortante basal, $V_{b}$, edificio EM2-I

Tabla 7. Cortantes basales y coeficientes sísmicos máximos obtenidos experimental y analíticamente

\begin{tabular}{ccccc}
\hline Ensaye No. & \multicolumn{2}{c}{ Ensaye } & $\begin{array}{c}\text { Cortante basal V } \\
(\mathbf{k N})\end{array}$ & $\begin{array}{c}\text { Coeficiente sísmico } \\
\text { máximo cmáx }\end{array}$ \\
\hline \multirow{2}{*}{ (1) } & M1-Exp & Ensaye & 8.15 & 0.123 \\
Int. baja & M1-Teo & Ruaumoko & 8.73 & 0.132 \\
& M1-Teo & ETABS & 8.74 & 0.132 \\
\hline \multirow{2}{*}{ Int. alta } & M2-Exp & Ensaye & 9.70 & 0.146 \\
& M2-Teo & Ruaumoko & 9.50 & 0.143 \\
M2-Teo & ETABS & 9.26 & 0.140 \\
\hline \multirow{2}{*}{ Int. alta } & M3-Exp & Ensaye & 8.76 & 0.132 \\
& M3-Teo & Ruaumoko & 9.10 & 0.137 \\
\hline
\end{tabular}

(1) $\mathrm{c}$ se define como el cociente del cortante basal y el peso de la superestructura.

\section{COMPARACIÓN DE LA RESPUESTA DINÁMICA: ESPECIMENES EM2 Y EM2-I}

A continuación, se comentan y comparan los resultados experimentales obtenidos durante los ensayes de intensidad alta del edificio con base fija EM2 (Blandón y Rodríguez, 2017), y el edificio con aisladores EM2-I. Dado que los resultados de los dos ensayes de intensidad alta (M2-Exp y M3-Exp), realizados para el edificio EM2-I fueron similares, en lo que sigue se considera sólo los resultados obtenidos en el ensaye M2-Exp.

\section{Propiedades dinámicas}

La tabla 8 compara las frecuencias y periodos obtenidos experimentalmente en los edificios EM2 y EM2-I. El periodo fundamental del edificio sin aislamiento, EM2, fue $0.485 \mathrm{~s}$, el valor del periodo fundamental del edificio EM2-I fue igual a $2.732 \mathrm{~s}$, valor igual a 5.6 veces el valor del periodo fundamental del edificio EM2. 
Tabla 8. Propiedades dinámicas: frecuencias y periodos (EM2 y EM2-I)

\begin{tabular}{ccccc}
\hline \multirow{2}{*}{ Modo } & \multicolumn{2}{c}{ EM2 $($ Exp) (sin aisladores) } & \multicolumn{2}{c}{ EM2-I (Exp) (con aisladores) } \\
\cline { 2 - 5 } & Frecuencia $(\mathbf{H z})$ & Periodo $(\mathbf{s})$ & Frecuencia $(\mathbf{H z})$ & Periodo $(\mathbf{s})$ \\
\hline 1 & 2.060 & 0.485 & 0.366 & 2.732 \\
2 & 7.309 & 0.137 & 3.052 & 0.328 \\
3 & 14.999 & 0.067 & 8.118 & 0.123 \\
4 & 21.851 & 0.046 & 14.954 & 0.067 \\
\hline
\end{tabular}

Con el empleo del espectro de respuesta de pseudoaceleración elástico se puede estimar el efecto en la respuesta del incremento del amortiguamiento y del periodo fundamental. La figura 25 muestra los espectros de respuesta elásticos de pseudoaceleración de la señal medida en la plataforma de la mesa vibradora en el ensaye M2-Exp, para valores de la fracción de amortiguamiento crítico, $\xi$, iguales a 3 y $15 \%$. Los resultados mostrados en la figura 25 muestran que el edificio EM2, sin aisladores, con periodo fundamental $T_{1}$ igual a $0.485 \mathrm{~s}$, se ubica en la zona de periodos con los mayores valores de ordenadas espectrales.

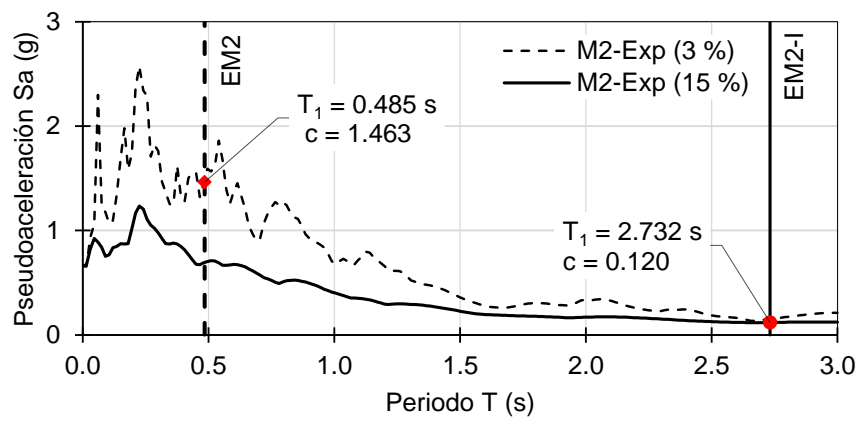

Figura 25. Espectros de respuesta elástico de pseudoaceleración ( $\xi$ igual a 3 y $15 \%$ )

Al utilizar el aislamiento de base en el espécimen, el periodo fundamental $T_{1}$ se incrementó a $2.732 \mathrm{~s}$, pasando a una zona de bajo contenido de frecuencias. De acuerdo con el espectro mostrado en la figura 25, los coeficientes sísmicos elásticos en el edificio EM2 y en el edificio EM2-I son iguales a 1.463 y 0.120 , respectivamente. Este último valor fue cercano a los valores obtenidos experimental y teóricamente.

\section{Reducción de aceleraciones de piso y distorsiones}

Los daños en elementos estructurales y no estructurales pueden ocurrir debido a las aceleraciones de piso, así como a las distorsiones globales y de entrepiso. Una de las ventajas del empleo del aislamiento de base es la reducción de estas aceleraciones y distorsiones. La figura 26a muestra los perfiles de desplazamientos relativos a la plataforma de la mesa vibradora divididos por la altura del edificio de ambos especímenes. Se observa que los desplazamientos máximos del edificio EM2-I, se concentraron en los aisladores. Esto debido la baja rigidez horizontal de estos elementos. Los desplazamientos máximos del edificio EM2, se presentaron en el último nivel. La figura 26b muestra la envolvente de distorsiones de entrepiso, $d r$, del edificio EM2 y EM2-I. Para el espécimen EM2-I, estas distorsiones se determinaron empleando los desplazamientos relativos al nivel N0.

En el ensaye de intensidad alta del edificio EM2 se obtuvo una distorsión global máxima, $D r_{\text {máx }}$, igual a $4 \%$, y una distorsión de entrepiso máxima, $d r_{\text {máx }}$, igual a $5.29 \%$. Estas distorsiones se redujeron de manera significativa en el espécimen aislado EM2-I, ya que en los ensayes de intensidad alta del edificio EM2-I, 
los valores de $D r_{\text {máx }}$ y $d r_{\text {máx }}$ fueron iguales a 0.63 y $0.97 \%$, respectivamente, bastante menores que las distorsiones medidas en el espécimen EM2. Estos resultados ilustran la importante reducción de demanda sísmica que se puede obtener en una estructura aislada respecto a la demanda en una estructura convencional sin aislamiento. Se debe notar que la planta baja tiene mayor altura de entrepiso y, por lo tanto, en ese nivel se presentó el valor máximo de la distorsión de entrepiso, $d r_{m a ́ x}$, en el espécimen.

a)

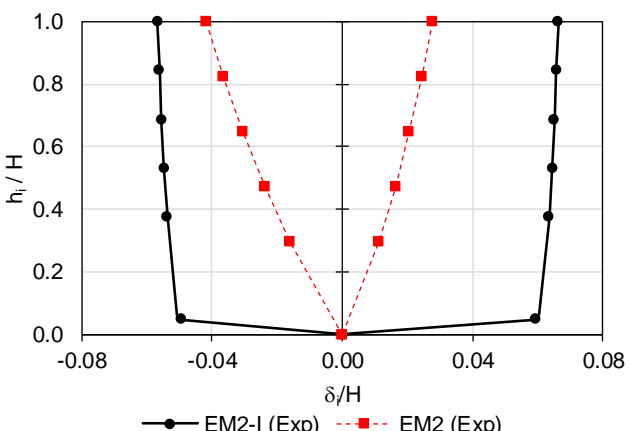

b)

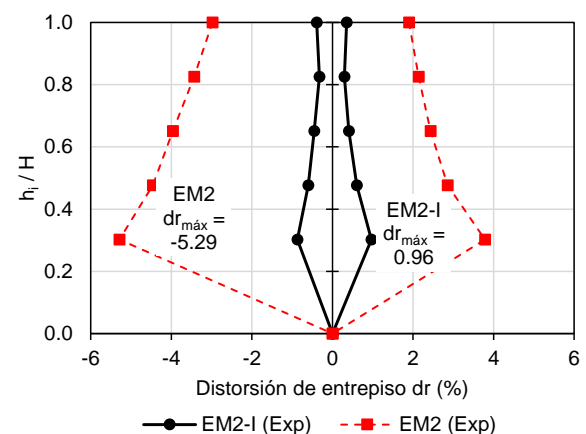

Figura 26. a) Envolvente de desplazamientos relativos, b) Distorsiones de entrepiso, $d r$

La figura 27a muestra las envolventes de aceleraciones absolutas de los diferentes pisos de ambos edificios. La máxima aceleración absoluta medida en el nivel N5 del edificio aislado EM2-I fue igual a $0.219 \mathrm{~g}$, lo cual representa el $16 \%$ de la máxima aceleración medida en el mismo nivel del edificio sin aislamiento EM2. La figura 27b muestra los espectros de piso de pseudoaceleraciones elásticas para el nivel N5 para los especímenes EM2 y EM-I, lo que indica una importante reducción de las aceleraciones de piso en el espécimen aislado.

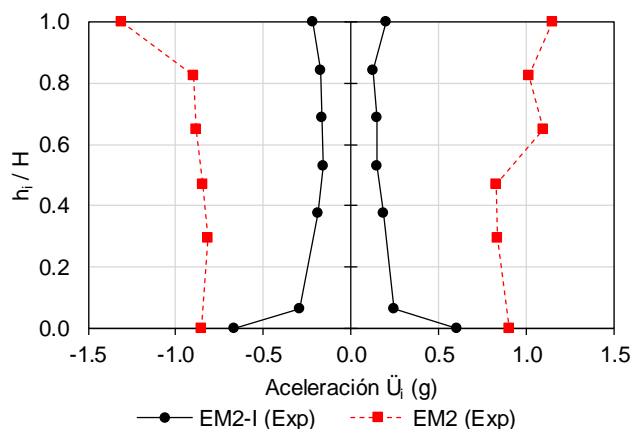

a)

Figura 27. a) Envolvente de aceleraciones medidas (EM2 y EM2-I), b) Espectro de piso de pseudoaceleraciones elástico de las señales medidas en el nivel N5 (EM2 y EM2-I)

La atenuación o amplificación de las aceleraciones pueden mostrarse como el cociente de las máximas aceleraciones medidas en el nivel N5 $\left(\ddot{U}_{5 \text { máx }}\right)$ y en la plataforma de la mesa vibradora $\left(\ddot{U}_{g m a ́ x}\right)$. En los ensayes de intensidad alta del edificio EM2, este cociente fue igual a 1.31, y para el edificio EM2-I, fue igual a 0.33. Estos resultados son congruentes con lo mostrado en la figura 27a, lo que indica una importante atenuación de las aceleraciones de piso en el edificio con aisladores, lo que se debe tanto al incremento del periodo fundamental, como al amortiguamiento. Estos resultados muestran la importante reducción de aceleraciones de piso y de distorsiones que es posible lograr en una estructura con aislamiento. 
El espectro de piso del espécimen EM2-I (figura 27b) muestra amplificación de aceleraciones en la zona de periodos cortos $(T<0.3 \mathrm{~s})$, lo cual se asocia a la respuesta del segundo modo de vibrar, esto debido a la no linealidad del sistema de aislamiento (Skinner et al., 1993).

\section{Reducción del coeficiente sísmico}

Una característica de la respuesta sísmica de estructuras que permite comparar la respuesta del edificio EM2-I y el edificio EM2, es el coeficiente sísmico, $c$, que se define como el cociente del cortante basal, $V_{b}$ y el peso de la superestructura $W_{s}$. Para determinar los valores de $c$ en el edificio EM2, se realizó un análisis estático no lineal (AENL) con cargas incrementales y distribución triangular, similar a la empleada en su diseño. De este análisis se obtuvo un coeficiente sísmico de fluencia, $c_{y}$ igual a 0.281 , y una distorsión global de fluencia, $D r_{y}$, igual a 1.2\%, figura 28a. Para este análisis, el valor de la distorsión global última, $D r_{u}$, se consideró igual a $5 \%$, para un coeficiente sísmico último, $c_{u}$ igual a 0.612 , figura 28 a. Como resultado del ensaye de intensidad alta del edificio sin aislamiento EM2, se obtuvo un coeficiente sísmico máximo, $c_{\text {máx }}$, igual a 0.55 , este valor superó al coeficiente sísmico de fluencia, $c_{y}$, por lo tanto, correspondió a la etapa de comportamiento inelástico, lo cual implica daño estructural. El edificio EM2 alcanzó una distorsión global máxima igual a $4 \%$, el cociente de este valor y el de fluencia indica que este edificio tuvo una ductilidad global de desplazamiento, $\mu_{d}$, igual a 3.3.

La figura 28a muestra la curva bilineal de resistencia lateral del edificio sin aislamiento EM2 obtenida del análisis estático no lineal ya descrito. Se indican los coeficientes sísmicos $c_{y}$ y $c_{\text {máx }}$, y las distorsiones globales $D r_{y}$ y $D r_{\text {máx }}$. Un criterio para verificar que el comportamiento de la superestructura del edificio EM2-I fue elástico durante los ensayes sísmicos es comparar los valores máximos del coeficiente sísmico y la distorsión global medidos en este espécimen, con los valores de fluencia del edificio EM2. La figura 28b muestra los valores del coeficiente sísmico, $c_{\text {máx }}$ y la distorsión global, $D r_{\text {máx }}$, medidos en el ensaye de intensidad baja M1-Exp y en el ensaye de intensidad alta M2-Exp. Durante los ensayes del espécimen EM2I, los coeficientes sísmicos, $c_{\text {máx }}$ medidos en los ensayes M1-Exp y M2-Exp fueron 0.123 y 0.146, respectivamente. Las distorsiones globales, $D r_{m a ́ x}$, asociadas a dichos coeficientes sísmicos fueron iguales a 0.45 y $0.63 \%$, para los ensayes mencionados. En ninguno de los ensayes en mesa vibradora se alcanzó los valores de fluencia de la superestructura, es decir el edificio de base aislada se comportó elásticamente, y por tanto los elementos estructurales no tuvieron algún tipo de daño.

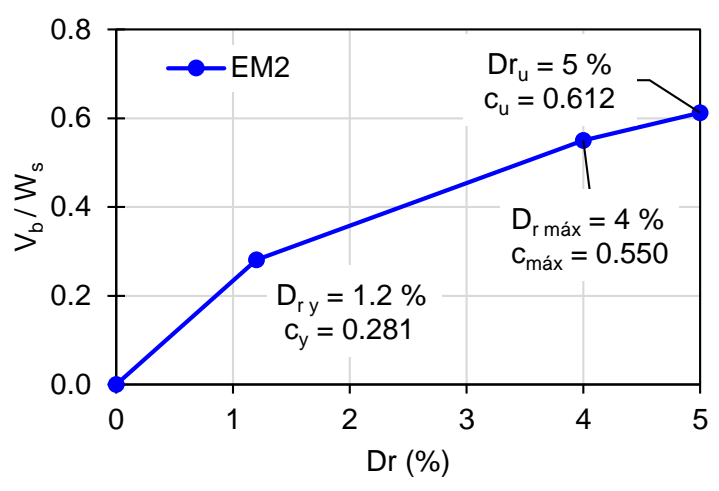

a)

Figura 28. Coeficientes sísmicos versus distorsión global. a) Espécimen EM2, b) Espécimen EM2-I

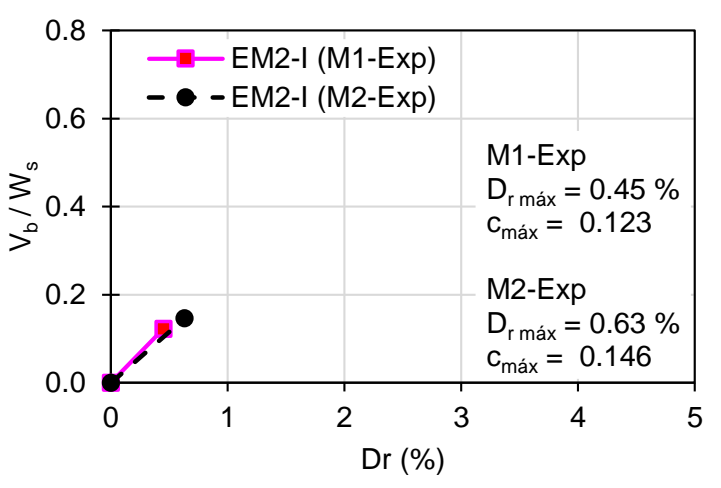

b) 


\section{RESUMEN DE RESULTADOS OBTENIDOS}

La tabla 9 muestra un resumen de datos importantes obtenidos teórica y experimentalmente en los ensayes de intensidad alta en el espécimen sin aislamientoEM2, y con aislamiento, EM2-I. Los valores experimentales del periodo fundamental y el amortiguamiento del edificio EM2-I fueron obtenidos durante el ensaye de intensidad alta M2-Exp, para el valor de desplazamiento máximo del aislador, $d_{\text {máx, }}$ igual a $176 \mathrm{~mm}$.

Tabla 9. Resumen de la comparación de la respuesta de los edificios EM2 y EM2-I

\begin{tabular}{|c|c|c|c|c|}
\hline \multirow{2}{*}{ Estructura } & \multicolumn{2}{|c|}{ EM2 } & \multicolumn{2}{|c|}{ EM2-I (M2-Exp) ${ }^{(1)}$} \\
\hline & Teórico & Experimental & Teórico & Experimental \\
\hline $\mathbf{W}_{\mathrm{s}}(\mathbf{k N})$ & 66.14 & 66.14 & 66.14 & 66.14 \\
\hline $\mathbf{W}(\mathbf{k N})^{(2)}$ & $-\ldots$ & $-\ldots$ & 79.24 & 79.24 \\
\hline $\mathbf{T}_{1}(\mathbf{s})$ & 0.501 & 0.485 & 2.732 & 2.732 \\
\hline$\xi(\%)$ & 3.0 & 2.0 & 15.0 & 15.1 \\
\hline$c_{y}$ & 0.281 & $-\ldots$ & 0.281 & $-\ldots$ \\
\hline $\mathbf{c}_{\mathbf{u}}$ & 0.612 & $--_{-}$ & 0.612 & - - - - \\
\hline $\mathbf{c}_{\text {máx }}$ & 0.630 & 0.550 & 0.143 & 0.146 \\
\hline Drmáx $(\%)$ & 3.20 & 4.00 & 0.35 & 0.63 \\
\hline drmáx $(\%)$ & 4.50 & 5.29 & 0.51 & 0.97 \\
\hline$\ddot{\mathbf{U}}_{\text {1máx }} / \ddot{\mathbf{U}}_{\text {gmáx }}$ & 0.93 & 0.87 & 0.32 & 0.28 \\
\hline$\ddot{\mathbf{U}}_{5 \text { máx }} / \ddot{\mathbf{U}}_{\text {gmáx }}$ & 1.45 & 1.31 & 0.37 & 0.33 \\
\hline$\ddot{\mathbf{U}}_{\text {gmáx }}$ & \multicolumn{2}{|c|}{0.90} & \multicolumn{2}{|c|}{0.66} \\
\hline
\end{tabular}

\section{CONCLUSIONES}

En esta investigación se llevó a cabo un estudio comparativo de resultados analíticos y experimentales de dos edificios estructurados con base en marcos de acero con fusibles en las zonas de posibles articulaciones plásticas. El primero, fue un edificio convencional de base fija de cinco niveles, denominado EM2 y ensayado en una investigación anterior (Blandón y Rodríguez, 2007). El segundo edificio, denominado EM2-I y ensayado en esta investigación, tuvo la misma superestructura que el mencionado espécimen de base fija, excepto por la adición de los aisladores en su base, los que fueron del tipo doble péndulo de fricción. Además, en el espécimen aislado se empleó un nivel adicional sobre el sistema de aislamiento, este nivel es identificado como N0. Ambos edificios fueron ensayados en mesa vibradora y fueron sometidos a demandas sísmicas comparables.

En el ensaye de intensidad baja, el sistema de aislamiento no participó en la respuesta dinámica, por lo que el comportamiento del edificio EM2-I correspondió al de una estructura de base fija. Esto se verificó mediante el cociente de aceleraciones absolutas medidas en el último nivel y en la plataforma de la mesa vibradora, $\ddot{U}_{5}$ y $\ddot{U}_{g}$, respectivamente, y mediante la historia de desplazamientos relativos de los aisladores medidos entre la placa superior e inferior del aislador. La respuesta obtenida durante este ensaye mostró un comportamiento típico de un edificio convencional. Este comportamiento se asoció a la baja intensidad de la señal, por lo que la fuerza de fricción no fue superada. Es importante señalar que el coeficiente sísmico máximo medido en este ensaye fue igual al $84 \%$ del coeficiente máximo medido en el ensaye de intensidad alta del edificio aislado EM2-I. 
El cambio de la respuesta dinámica del edificio EM2-I, ocurrió en los ensayes de intensidad alta. En estos ensayes el sistema de aislamiento mejoró la respuesta de manera considerable. Se incrementó el periodo fundamental 5.6 veces respecto al periodo fundamental del edificio EM2, y el amortiguamiento efectivo obtenido fue igual a $15 \%$. El comportamiento del espécimen EM2-I fue comparable al de un cuerpo rígido oscilando sobre el sistema de aislamiento, reduciendo las distorsiones de entrepiso y la distorsión global respecto a las del edificio sin aislar. En lo que sigue se resumen algunos resultados relevantes obtenidos en los ensayes del espécimen EM2-I.

- La máxima aceleración absoluta medida en el nivel N5 fue igual al $33 \%$ de la máxima aceleración absoluta medida en la plataforma de la mesa vibradora.

- $\quad$ La máxima distorsión global medida fue $0.63 \%$.

- La máxima distorsión de entrepiso medida fue $0.97 \%$, la que ocurrió en el nivel N1. Las máximas distorsiones de entrepiso medidas en los pisos superiores fueron cercanas a uno de los valores límites especificado por la NTCS (2017) para el control de daños ante sismos frecuentes.

Se deben mencionar las siguientes mejoras en la respuesta dinámica observada en el espécimen EM2I respecto al edificio de base fija EM2.

- El máximo coeficiente sísmico medido fue $26 \%$ del valor máximo de este coeficiente medido en el espécimen EM2.

- La máxima distorsión global medida fue $16 \%$ del valor de distorsión la máxima medida en el espécimen EM2.

- La máxima distorsión de entrepiso medida fue 18 \% del valor de la distorsión máxima medida en el espécimen EM2.

Los resultados encontrados en esta investigación permitieron demostrar las ventajas del empleo de aislamiento en estructuras, en particular el empleo de aisladores de doble péndulo de fricción, como alternativa de diseño sismorresistente para mejorar de manera significativa el desempeño estructural de edificaciones ante acciones sísmicas.

\section{AGRADECIMIENTOS}

Esta investigación fue patrocinada por CONACyT, Proyecto de Investigación No 167445, por lo que se agradece a CONACyT este patrocinio. El proyecto experimental fue desarrollado en la Mesa Vibradora del Instituto de Ingeniería, UNAM. Los aisladores fueron donados por FIP Industriale, (Italia). Esta empresa construyó los aisladores de doble péndulo de fricción requeridos y realizó los ensayes dinámicos de los aisladores en su laboratorio. Se agradece a FIP Industriale por su importante colaboración para llevar a cabo esta investigación en México. También se agradece a Maria G. Castellano y Samuele Infanti, Investigadora y Jefe de Laboratorio de FIP MEC, respectivamente, por sus contribuciones que permitieron mejorar la calidad de esta investigación. Asimismo, se agradece a la firma de consultoría en sistemas de aislamiento sísmico, Protección Sísmica de México por sus comentarios aportados en el desarrollo de esta investigación. El primer autor agradece a CONACyT por la beca otorgada para cursar los estudios de Posgrado.

\section{REFERENCIAS}

Applied Tecnology Council (ATC), (2017), "Seismic Analysis, Design, and Installation of Nonstructural Components and Systems- Background and Recommendations for Future Work, NIST GCR 17917-44. https://doi.org/10.6028/NIST.GCR 17-917-44. 
ASCE/SEI 7-16 (2017), "Minimum design loads and associated criteria for buildings and other structures", American Society of Civil Engineers, Structural Engineering Institute.

Blandón, J y Rodríguez, M (2007), "Estudio analítico-experimental y propuesta de diseño sísmico de sistemas de pisos rígidos en edificios", Series de Investigación y Desarrollo, SID/656, Instituto de Ingeniería, UNAM.

Carr, A (1998), "Ruaumoko 2D”, Computer Program Library, Universidad de Canterbury, Departamento de Ingeniería Civil.

Comisión Federal de Electricidad, Instituto de Investigaciones Eléctricas, (2015), "Manual de Diseño de Obras Civiles, Diseño por Sismo", Capítulo C.1.3: Diseño por Sismo.

Computers and Structures, Inc. (1984-2016), "ETABS 2016 Integrated Building Design Software", Computers and Structures, Berkeley, California, EUA.

Constantinou, M, Mokha, A y Reinhorn, A M (1990), "Teflon Bearings in Base Isolation II: Modeling”, Journal of Structural Engineering, ASCE, Vol 116, Issue 2.

EN 15129 (2009), "European Standard Anti-seismic Devices”, Commitee for Standardization (CEN).

McVitty, W J y Constantinou, M C (2015), "Property Modification Factors for Seismic Isolators: Design Guidance for Buildings", Technical Report MCEER-15-0005.

Naeim, F y Kelly, J (1999), "Design of Seismic Isolated Structures", John Wiley \& Sons, INC. New York. NTCS-2017, Norma Técnica Complementaria por Sismo (2017). Gaceta oficial de la Ciudad de México.

Skinner, R I, Robinson, W H y McVerry, G H (1993), “An Introduction to Seismic Isolation”, John Wiley and Sons Ltd, Baffins Lane, Chichester, England.

Ordaz, M y Montoya, C (2012), “DEGTRA ver. 9.1”, Instituto de Ingeniería, UNAM.

Ponzo, F C, Di Cesare, A, Nigro, D, Simonetti, M y Lecceses, G (2014), "Shaking table tests of a base isolated structure with doublé concave friction pendulums", New Zealand Society for Earthquake Engineering, Conference.

Restrepo, JI, Rodriguez, M, Kao-Stocker, G, Carr, AJ (2014), "Seismic Response of a Four-Story Miniature Building with Replaceable Plastic Hinges", Journal of Earthquake Engineering, 18:8, 1217-1240

Rodríguez, M E (2020), Zenodo: http://doi.org/10.5281/zenodo.3606726

U.S. Geological Survey (1996), "USGS Response to an Urban Earthquake: Northridge '94", Federal Emergency Management Agency (FEMA). 